\title{
POWER SPECTRA OF RANDOM SPIKE FIELDS AND RELATED PROCESSES
}

\author{
PIERRE BRÉMAUD, ${ }^{*}$ École Polytechnique Fédérale de Lausanne and INRIA-ENS \\ LAURENT MASSOULIÉ,** Microsoft Research \\ ANDREA RIDOLFI, ${ }^{* * *}$ École Polytechnique Fédérale de Lausanne
}

\begin{abstract}
In this article, we review known results and present new ones concerning the power spectra of large classes of signals and random fields driven by an underlying point process, such as spatial shot noises (with random impulse response and arbitrary basic stationary point processes described by their Bartlett spectra) and signals or fields sampled at random times or points (where the sampling point process is again quite general). We also obtain the Bartlett spectrum for the general linear Hawkes spatial branching point process (with random fertility rate and general immigrant process described by its Bartlett spectrum). We then obtain the Bochner spectra of general spatial linear birth and death processes. Finally, we address the issues of random sampling and linear reconstruction of a signal from its random samples, reviewing and extending former results.
\end{abstract}

Keywords: Shot noise; random sampling; point process; Bochner power spectral measure; Bartlett power spectral measure; Hawkes process; random field; branching process; linear birth-death process

2000 Mathematics Subject Classification: Primary 60G12; 60G35; 62M15; 62M40;

$60 \mathrm{G} 55 ; 60 \mathrm{G} 60 ; 60 \mathrm{~J} 27 ; 60 \mathrm{~J} 80$

Secondary 60G20; 62M $30,94 \mathrm{~A} 05 ; 94 \mathrm{~A} 20$

\section{Introduction}

\subsection{Classes of signals related to point processes}

This article is concerned with the second-order properties of 'signals' (stochastic processes) related to random spike fields, that is, spatial point processes. More specifically, we shall consider three types of signal:

(a) the random spike fields themselves;

(b) the filtered random spike fields;

(c) the modulated random spike fields.

These types of signal are depicted in Figure 1 in the one-dimensional case (spike fields are then called spike trains or Dirac combs). Signals of the second category are also known as shot noises, and the third category arises, in particular, in random sampling. Signals of the first

Received 22 June 2004; revision received 27 June 2005.

* Postal address: INRIA-ENS, Département d'Informatique, École Normale Supérieure, 45 rue d’Ulm, F-75005 Paris, France. Email address: pierre.bremaud@ens.fr

** Postal address: Microsoft Research, 7 J. J. Thomson Avenue, Cambridge CB3 0FB, UK.

*** Postal address: School of Computer and Communication Sciences, École Polytechnique Fédérale de Lausanne, CH-1015 Lausanne, Switzerland. 

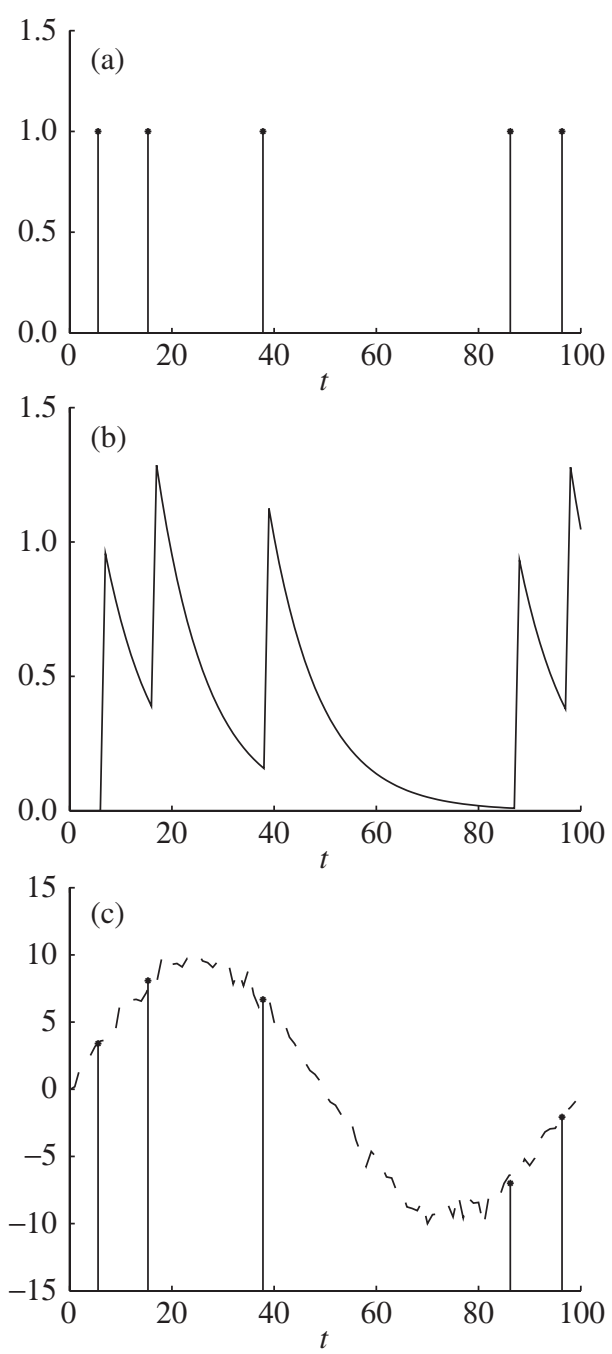

FIGURE 1: Random spike train and related processes. (a) Random spike train (point process). (b) Filtered spike train (shot noise). (c) Modulated spike train (randomly located samples).

category, point processes, form the basic element on which the other signals of interest in this research/review article are constructed.

In this article, we give the power spectra (sometimes in a generalized sense) for the above three categories of signal. This is done in quite general cases; in particular, concerning shot noises, cluster point processes, jittered point processes, and Hawkes branching point processes, we do not require the basic point process to be either a homogeneous Poisson process, a renewal process, or a Cox process.

Shot noises have received much attention in the applied literature, both in physics and in electrical engineering. They model thermionic noise in conductors [29] (also see the references in [6]); queuing systems, for instance in the form of $\mathrm{M} / \mathrm{GI} / \infty$ pure delay systems and traffic flows in communications systems; and delayed claims in insurance risk analysis [17], [28]. The 
signals arising in neurophysiology are typically non-Poisson shot noises and the interference field in a mobile communications system is aptly modeled as a spatial shot noise (see, for instance, [3]). Shot noises also arise naturally in wavelet signal analysis when the analyzed signal is a point process, since in this case the wavelet coefficients are samples of shot noises. Wavelet statistical analysis has been proposed to detect and compute the Hurst parameter in classical signals and the method applies equally well to random Dirac combs with long-range dependence properties [1], [2]. For references on clustered point processes and jittered point processes, the reader is referred to [14]. In the present article, they are considered as special cases of shot noises (the impulse function being replaced by a point process measure).

A modulated Dirac comb is a Dirac comb with pulses of varying height. In random sampling, the height of a pulse is equal to the value of the signal sampled at that time. Random sampling has been extensively studied in view of spectral analysis, the object being to recover the power spectrum of the signal from the modulated sample comb, or even from the sample sequence (without timing information); a specific domain of application is laser velocimetry, where the samples are collected only at the passage of a reflecting particle through the laser beam. Early investigation of random sampling [30] was mostly motivated by the search for alias-free sampling schemes, that is, sampling schemes leading to a one-to-one relation between the spectrum of the sample comb and that of the sampled signal. The first detailed analyses of randomly sampled signals were based on the modeling of the sample comb using the Dirac (pseudo)process $\delta$. In [4], [18], and [5], Beutler and Leneman obtained formulae for the moments of the sample comb that led to the expression of the correlation of the sample comb as a function of the correlation of the sampled signal. Leneman and Lewis [19] investigated the reconstruction error for several interpolators of random samples. Such results depend on the sampling scheme through statistics related to the intervals between successive points of the sampler (i.e. the point process). Modulated random spike fields are studied in Section 5.

As for the spike fields themselves, we recall the basic theory of Bartlett spectra in Section 2. The Hawkes branching point processes are studied in Section 4. Hawkes processes were introduced, under the name of self-exciting point processes, in [15] and further studied in [16]; also see [14]. Such branching point processes are of interest in epidemics and in seismology (see [31]), where they are known as epidemic-type aftershock models [24].

The generalized linear (not necessarily Markovian) birth-death processes are shot noises for which the basic point process is a Hawkes process. Note that such a process can be viewed as a shot noise on a Hawkes process, and the results of Section 3 do not apply since in this case the 'shots' are not independent of the basic point process.

\subsection{Reviewed results and novel results}

The present paper can be considered partly as a review, sometimes with (mostly trivial) extensions to the spatial case. For instance, the results on clustered point processes and jittered point processes appear in [14] (in Example 8.2(d) and Exercise 8.2.6, respectively), and those on modulated point processes appear in [14, Example 8.4(c)] and, previously, in the seminal papers of [21] and [20]. However, this article is not an exhaustive review of the subject; rather, we concentrate on the actual computation of the spectra of complex signals, the motivation for this work being applications to ultrawide-band communications and multipath fading channels (see the dissertation [25], as well as the articles [26] and [27]). For more exhaustive reviews, the reader is referred to [11], [10], and [12], and, of course, to [14].

We present the results on shot noises, cluster point processes, and jittered point processes in a unified manner, showing that they can be derived from a single formula (the fundamental 
isometry formula), which appears here for the first time. The conditions of validity of this formula are most important, in that they allow a precise description of the test functions used in the definition of the Bochner or Bartlett spectra of the processes considered.

Another new result concerns the Bartlett spectrum of Hawkes branching point processes with random fertility rates and general ancestor point processes. Earlier results in this direction can be found in [9]. (Note, however, that the method of [9] does not work.) Also see [8], where the critical case, leading to long-range dependence, was considered. Note that although Hawkes processes are a particular case of cluster point processes, their spectra cannot be obtained easily from the general formula for cluster point processes recalled in Section 3. Our analysis is more direct than that of [9], and it allows us to obtain more insight into the test functions used in the definition of the Bartlett spectrum, as already mentioned.

Among the new results, we obtain the Cramér spectrum of the generalized spatial birth and death processes under the same general conditions (i.e. with a general birth process and general lifetimes) as for the Hawkes processes.

Concerning modulated point processes, our contribution does not go far beyond the original results of [21] and [20] (and the presentation given in [14]). There, the spectrum of the sample sequence was expressed as a function of the spectrum of the sampled signal and of the secondorder quantities of the point process, and then, by reformulating the concept of being alias free, alias-free sampling schemes were proved that led to a consistent spectral estimator. This work is closest in content to ours; our method of proof is the same as in [14], our contribution being to give more details of the proof there (given in Example 8.4(c)). These details also turn out to be useful in determining the class of test functions for which the defining formula of the spectrum is true. The novel results in this article concerning modulated point processes are an expression for the power spectra of modulated spike fields when the sampler is possibly dependent on the signal, and, in the independent case, an expression for the error when the signal is approximated by a filtered version of the samples; that is, the reconstruction error.

\section{Covariance and spectral measure}

\subsection{The covariance measure}

Let $N$ be a simple and locally bounded point process on $\mathbb{R}^{m}$. It is called a second-order point process if, for all bounded Borel sets $C \subset \mathbb{R}^{m}$,

$$
\mathrm{E}\left[N(C)^{2}\right]<\infty .
$$

The formula

$$
v(C)=\mathrm{E}[N(C)]
$$

defines a Radon (that is, locally finite) measure $v$ on $\mathbb{R}^{m}$ called the mean measure or intensity measure of $N$. By Campbell's theorem, for all measurable functions $\varphi: \mathbb{R}^{m} \rightarrow \mathbb{R}$ that are nonnegative or belong to $L_{\mathbb{C}}^{1}(v)$, the sum $\sum_{t \in N} \varphi(t)=N(\varphi)$ is well defined and

$$
\mathrm{E}[N(\varphi)]=\mathrm{E}[v(\varphi)]
$$

Moreover, the measure $M_{2}$ on $\mathbb{R}^{m} \times \mathbb{R}^{m}$ defined by

$$
M_{2}(A \times B)=\mathrm{E}[N(A) N(B)]
$$

is a Radon measure. 
Definition 1. By definition, $L_{N}^{2}\left(M_{2}\right)$ is the collection of measurable functions $\varphi: \mathbb{R}^{m} \rightarrow \mathbb{C}$ such that

$$
\mathrm{E}\left[N(|\varphi|)^{2}\right]<\infty
$$

which implies that $\varphi \in L_{\mathbb{C}}^{1}(v)$.

Clearly, $L_{N}^{2}\left(M_{2}\right)$ is a vector space that contains all bounded functions with compact support, and, if $\varphi, \psi \in L_{N}^{2}\left(M_{2}\right)$, we have

$$
\mathrm{E}\left[\left(\int_{\mathbb{R}^{m}} \varphi(t) N(\mathrm{~d} t)\right)\left(\int_{\mathbb{R}^{m}} \psi(t) N(\mathrm{~d} t)\right)^{*}\right]=\int_{\mathbb{R}^{m}} \int_{\mathbb{R}^{m}} \varphi(t) \psi^{*}(s) M_{2}(\mathrm{~d} t \times \mathrm{d} s) .
$$

We now assume that $N$ is of second order and stationary, in which case

$$
v(C)=\lambda \ell^{m}(C)
$$

for some $\lambda \in \mathbb{R}_{+}$called the intensity (where $\ell^{m}$ is the Lebesgue measure on $\mathbb{R}^{m}$ ). By a previous remark, it follows that

$$
L_{N}^{2}\left(M_{2}\right) \subseteq L_{\mathbb{C}}^{1}\left(\mathbb{R}^{m}\right)
$$

By stationarity again, for all Borel sets $A, B \subseteq \mathbb{R}^{m}$ and all $t \in \mathbb{R}^{m}$, we have

$$
M_{2}((A+t) \times(B+t))=M_{2}(A \times B) .
$$

It follows from [14, Lemma A2.7.II, p. 409] that, for all $\varphi, \psi \in L_{N}^{2}\left(M_{2}\right)$,

$$
\int_{\mathbb{R}^{m}} \int_{\mathbb{R}^{m}} \varphi(t) \psi^{*}(s) M_{2}(\mathrm{~d} t \times \mathrm{d} s)=\int_{\mathbb{R}^{m}}\left(\int_{\mathbb{R}^{m}} \varphi(t) \psi^{*}(s+t) \mathrm{d} t\right) \sigma(\mathrm{d} s)
$$

for some Radon measure $\sigma$, and from (1) and (2), for $\varphi, \psi \in L_{N}^{2}\left(M_{2}\right)$ we have

$$
\operatorname{cov}\left(\int_{\mathbb{R}^{m}} \varphi(t) N(\mathrm{~d} t), \int_{\mathbb{R}^{m}} \psi(s) N(\mathrm{~d} s)\right)=\int_{\mathbb{R}^{m}}\left(\int_{\mathbb{R}^{m}} \varphi(t) \psi^{*}(t+s) \mathrm{d} t\right) \Gamma_{N}(\mathrm{~d} s),
$$

where the Radon measure

$$
\Gamma_{N}:=\sigma-\lambda^{2} \ell^{m}
$$

is called the covariance measure of the stationary second-order point process $N$.

\subsection{The spectral measure}

Definition 2. Let $N$ be a simple, stationary second-order point process on $\mathbb{R}^{m}$ with intensity $\lambda$. Let $B_{N}$ be a vector space of functions such that $B_{N} \subseteq L_{N}^{2}\left(M_{2}\right)$. A measure $\mu_{N}$ on $\mathbb{R}^{m}$ is called the Bartlett spectral measure of $N$ on the domain $B_{N}$ if, for all $\varphi \in B_{N}$, the identity

$$
\operatorname{var}\left(\int_{\mathbb{R}^{m}} \varphi(t) N(\mathrm{~d} t)\right)=\int_{\mathbb{R}^{m}}|\hat{\varphi}(v)|^{2} \mu_{N}(\mathrm{~d} \nu)
$$

(where $\hat{\varphi}(v)=\int_{\mathbb{R}^{m}} \mathrm{e}^{-2 \mathrm{i} \pi\langle v, t\rangle} \mathrm{d} t,\langle\cdot, \cdot\rangle$ being the scalar product) holds, the two terms of the equality being finite. The space $B_{N}$ is also called a test function space for the point process $N$. 
By polarization of (3), for all $\varphi, \psi \in B_{N}$ we have

$$
\operatorname{cov}(N(\varphi), N(\psi))=\int_{\mathbb{R}^{m}} \hat{\varphi}(v) \hat{\psi}^{*}(v) \mu_{N}(\mathrm{~d} v) .
$$

A suitable space $B_{N}$ of test functions will be determined in each situation. Ideally, we want to determine the largest possible domain $B_{N}$. We are looking for conditions of the form $\varphi \in L_{\mathbb{C}}^{1}\left(\mathbb{R}^{m}\right) \cap L_{\mathbb{C}}^{2}\left(\mathbb{R}^{m}\right)$, for instance. Note that $\varphi$ is necessarily contained in $L_{\mathbb{C}}^{1}\left(\mathbb{R}^{m}\right)$ since, as observed earlier, $L_{N}^{2}\left(M^{2}\right) \subseteq L_{\mathbb{C}}^{1}\left(\mathbb{R}^{m}\right)$. In particular, the Fourier transform of any $\varphi \in B_{N}$ is well defined.

The existence and uniqueness of the Bartlett spectrum is the content of Theorem 1, which can be found in [23]. The theorem also shows that it is always possible to take for $B_{N}$ the space of functions that, together with their Fourier transforms, are $O\left(1 /|t|^{2}\right)$ as $|t| \rightarrow \infty$. We introduce the notation $\breve{u}(t)=u(-t)$ for functions.

Theorem 1. (Neveu [23].) Let $N$ be a stationary second-order point process and let $\sigma$ be the corresponding Radon measure, as in (2). There exists a unique nonnegative Radon measure $\hat{\sigma}$ on $\left(\mathbb{R}^{m}, \mathcal{B}\left(\mathbb{R}^{m}\right)\right)$ such that if $f$ and its Fourier transform are $O\left(1 /|t|^{2}\right)$ as $|t| \rightarrow \infty$, then

$$
\int_{\mathbb{R}^{m}} f(v) \hat{\sigma}(\mathrm{d} v)=\int_{\mathbb{R}^{m}} \hat{f}(t) \sigma(\mathrm{d} t)
$$

and, if $g$ satisfies the same conditions as $f$,

$$
\mathrm{E}[N(f) N(g)]=\lambda \int_{\mathbb{R}^{m}} \hat{f}(v) \check{\hat{g}}(v) \hat{\sigma}(\mathrm{d} v) .
$$

Theorem 8.6.III of [14] contains a general condition on the test functions. However, it is not explicit, since it is stated in terms of the spectral measure ( $f$ is required to be integrable with respect to $\sigma$, with a Fourier transform that is integrable using the Bartlett spectral measure). One of the purposes of the present article is to find explicit spaces of test function, and to show how these are modified by the various transformations that we study.

Example 1. (Regular grid.) Consider the point process on $\mathbb{R}^{2}$ whose points form a regular $\left(T_{1}, T_{2}\right)$-grid on $\mathbb{R}^{2}$ with random origin, that is,

$$
N=\left\{\left(n_{1} T_{1}+U_{1}, n_{2} T_{1}+U_{2}\right),\left(n_{1}, n_{2}\right) \in \mathbb{Z}^{2}\right\}
$$

where $T_{1}>0, T_{2}>0$, and $U_{1}$ and $U_{2}$ are independent uniform random variables on [0, $\left.T_{1}\right]$ and $\left[0, T_{2}\right]$, respectively. The point process is obviously stationary and second order, with average intensity $\lambda=1 /\left(T_{1} T_{2}\right)$. Its Bartlett spectral measure is

$$
\mu_{N}=\frac{1}{T_{1}^{2} T_{2}^{2}} \sum_{\left(n_{1}, n_{2}\right) \neq(0,0)} \varepsilon_{\left(n_{1} / T_{1}, n_{2} / T_{2}\right)},
$$

where $\varepsilon_{a}$ is the Dirac measure at $a$, and we can take

$$
B_{N}=\left\{\varphi: \varphi \in L_{\mathbb{C}}^{1}\left(\mathbb{R}^{2}\right), \sum_{n_{1}, n_{2} \in \mathbb{Z}}\left|\hat{\varphi}\left(\frac{n_{1}}{T_{1}}, \frac{n_{2}}{T_{2}}\right)\right|<\infty\right\}
$$


Let us prove (4). Equation (5) guarantees that the weak Poisson formula holds. More precisely (see, for instance, [7, Theorem A2.3, pp. 28]), the left-hand side of the equality

$$
\sum_{n_{1}, n_{2} \in \mathbb{Z}} \varphi\left(u_{1}+n_{1} T_{1}, u_{2}+n_{2} T_{2}\right)=\frac{1}{T_{1} T_{2}} \sum_{n_{1}, n_{2} \in \mathbb{Z}} \hat{\varphi}\left(\frac{n_{1}}{T_{1}}, \frac{n_{2}}{T_{2}}\right) \exp \left(2 \mathrm{i} \pi\left(\frac{n_{1}}{T_{1}} u_{1}+\frac{n_{2}}{T_{2}} u_{2}\right)\right)
$$

is well defined, and the equality holds for almost all $\left(u_{1}, u_{2}\right) \in \mathbb{R}^{2}$ (with respect to the Lebesgue measure). By (6),

$$
\begin{aligned}
\int_{\mathbb{R}^{2}} \varphi(t) N(\mathrm{~d} t) & =\sum_{n_{1}, n_{2} \in \mathbb{Z}} \varphi\left(U_{1}+n_{1} T_{1}, U_{2}+n_{2} T_{2}\right) \\
& =\frac{1}{T_{1} T_{2}} \sum_{n_{1}, n_{2} \in \mathbb{Z}} \hat{\varphi}\left(\frac{n_{1}}{T_{1}}, \frac{n_{2}}{T_{2}}\right) \exp \left(2 \mathrm{i} \pi\left(\frac{n_{1}}{T_{1}} U_{1}+\frac{n_{2}}{T_{2}} U_{2}\right)\right) .
\end{aligned}
$$

The rest of the proof is straightforward, noting that the finiteness of the sum in (6) implies

$$
\sum_{n_{1}, n_{2} \in \mathbb{Z}}\left|\hat{\varphi}\left(\frac{n_{1}}{T_{1}} u_{1}, \frac{n_{2}}{T_{2}} u_{2}\right)\right|^{2}<\infty .
$$

Example 2. (Cox process.) Let $N$ be a Cox point process on $\mathbb{R}^{m}$ with stochastic intensity $\{\lambda(t)\}_{t \in \mathbb{R}^{m}}$. By this we mean the following. First, $\{\lambda(t)\}_{t \in \mathbb{R}^{m}}$ is a nonnegative, almost surely locally integrable process and, second, conditionally on this process, $N$ is a Poisson process with intensity $\lambda(t)$. We suppose that $\{\lambda(t)\}_{t \in \mathbb{R}^{m}}$ is a wide-sense stationary process with mean $\lambda$ and Bochner spectral measure $\mu_{\lambda}$. Then the Bartlett spectrum of $N$ is

$$
\mu_{N}(\mathrm{~d} v)=\mu_{\lambda}(\mathrm{d} v)+\lambda \mathrm{d} v
$$

with domain $B_{N}=L_{\mathbb{C}}^{1}\left(\mathbb{R}^{m}\right) \cap L_{\mathbb{C}}^{2}\left(\mathbb{R}^{m}\right)$. Moreover, in this case this is the maximal domain, since $B_{N}=L_{N}^{2}\left(M_{2}\right)$. We omit the proof of (7), the details of which can be found in [25].

\section{Basic isometry formula}

\subsection{Filtered point process fields}

Consider a marked point process on $\mathbb{R}^{m}$ with marks in the measurable space $(K, \mathcal{K})$. Let $N$ be its basic point process on $\mathbb{R}^{m}$, assumed locally finite and simple, and let $\{Z(t)\}_{t \in \mathbb{R}^{m}}$ be its mark process. Assume that the random variables $Z(t)$ are independent and identically distributed, with common probability distribution $Q$, and are independent of $N$. Also assume that $N$ is a stationary second-order point process with Bartlett spectral measure $\mu_{N}$ on the domain $B_{N}$.

Let $Z$ be a random element with distribution $Q$. We introduce (or recall) the notation $L_{\mathbb{C}}^{p}(\ell \times Q)$ for the set of functions $\varphi: \mathbb{R}^{m} \times K \rightarrow \mathbb{C}$ such that

$$
\int_{\mathbb{R}^{m}} \mathrm{E}\left[|\varphi(t, Z)|^{p}\right] \mathrm{d} t<\infty
$$

In particular, $\varphi(t, Z) \in L_{\mathbb{C}}^{p}(\mathrm{P})$, that is, $\mathrm{E}\left[|\varphi(t, Z)|^{p}\right]<\infty$, for almost all $t \in \mathbb{R}$ (with respect to the Lebesgue measure). 
Let $\varphi: \mathbb{R}^{m} \times K \rightarrow \mathbb{R}$ be a measurable function such that

$$
\varphi \in L_{\mathbb{C}}^{1}(\ell \times Q) .
$$

For almost all $t$, we can define

$$
\bar{\varphi}(t)=\mathrm{E}[\varphi(t, Z)] .
$$

It follows from (8) that $\bar{\varphi} \in L_{\mathbb{C}}^{1}\left(\mathbb{R}^{m}\right)$ and, for $Q$-almost all $z \in K$, that $\varphi(\cdot, z) \in L_{\mathbb{C}}^{1}\left(\mathbb{R}^{m}\right)$. Let the Fourier transforms of these two functions be denoted by $\hat{\bar{\varphi}}$ and $\hat{\varphi}(\cdot, z)$, respectively. Moreover, suppose that

$$
\varphi \in L_{\mathbb{C}}^{2}(\ell \times Q) .
$$

Condition (9) implies that $\int_{\mathbb{R}^{m}}|\mathrm{E}[\varphi(t, Z)]|^{2} \mathrm{~d} t<\infty$, that is $\bar{\varphi} \in L_{\mathbb{C}}^{2}\left(\mathbb{R}^{m}\right)$, and, for $Q$-almost all $z \in K$, that $\varphi(\cdot, z) \in L_{\mathbb{C}}^{2}\left(\mathbb{R}^{m}\right)$. Observe that

$$
\hat{\bar{\varphi}}(v)=\mathrm{E}[\hat{\varphi}(v, Z)]=: \overline{\hat{\varphi}}(v)
$$

Finally, suppose that

$$
\bar{\varphi} \in B_{N}
$$

We can now state a fundamental isometry formula.

Theorem 2. Let $N$ and $\{Z(t)\}_{t \in \mathbb{R}^{m}}$ be as defined above, and let $\varphi, \psi: \mathbb{R}^{m} \times K \rightarrow \mathbb{R}$ satisfy (8), (9), and (10). Then

$$
\begin{aligned}
\operatorname{cov}( & \left.\sum_{t \in N} \varphi(t, Z(t)), \sum_{t \in N} \psi(t, Z(t))\right) \\
& =\int_{\mathbb{R}^{m}} \hat{\bar{\varphi}}(v) \hat{\bar{\psi}}^{*}(v) \mu_{N}(\mathrm{~d} v)+\lambda \int_{\mathbb{R}^{m}} \operatorname{cov}\left(\hat{\varphi}(v, Z), \hat{\psi}^{*}(v, Z)\right) \mathrm{d} v,
\end{aligned}
$$

where $Z$ is a $K$-valued random variable with distribution $Q$.

Proof. Formally,

$$
\begin{aligned}
& \mathrm{E}\left[\left(\sum_{t \in N} \varphi(t, Z(t))\right)\left(\sum_{t \in N} \psi(t, Z(t))\right)\right] \\
& \quad=\mathrm{E}\left[\sum_{t, t^{\prime} \in N, t \neq t^{\prime}} \varphi(t, Z(t)) \psi\left(t^{\prime}, Z\left(t^{\prime}\right)\right)\right]+\mathrm{E}\left[\sum_{t \in N} \varphi(t, Z(t)) \psi^{*}(t, Z(t))\right] \\
& =\mathrm{E}\left[\sum_{t, t^{\prime} \in N, t \neq t^{\prime}} \bar{\varphi}(t) \bar{\psi}^{*}\left(t^{\prime}\right)\right]+\mathrm{E}\left[\sum_{t \in N} \varphi(t, Z) \psi^{*}(t, Z)\right] \\
& =\mathrm{E}\left[\left(\sum_{t \in N} \bar{\varphi}(t)\right)\left(\sum_{t^{\prime} \in N} \bar{\psi}^{*}\left(t^{\prime}\right)\right)\right]-\mathrm{E}\left[\sum_{t \in N} \bar{\varphi}(t) \bar{\psi}^{*}(t)\right]+\mathrm{E}\left[\sum_{t \in N} \varphi(t, Z) \psi^{*}(t, Z)\right] \\
& =: a-b+c .
\end{aligned}
$$

These computations are justified because the three terms $a, b$, and $c$ are finite when $\varphi$ and $\psi$ are replaced by their absolute values. This follows from Schwarz's inequality and the facts 
that (for $a$ and $b$ ) $\bar{\varphi}$ and $\bar{\psi}$ are in both $L_{N}^{2}\left(M_{2}\right)$ and $L_{\mathbb{C}}^{2}\left(\mathbb{R}^{m}\right)$ and (for $c$ ) (9) holds. Since $\mathrm{E}\left[\sum_{t \in N} \varphi(t, Z(t))\right]=\mathrm{E}\left[\sum_{t \in N} \bar{\varphi}(t)\right]$, we have

$$
\begin{aligned}
& \operatorname{cov}\left(\sum_{t \in N} \varphi(t, Z(t)), \sum_{t \in N} \psi(t, Z(t))\right) \\
& \quad=\operatorname{cov}\left(\sum_{t \in N} \bar{\varphi}(t), \sum_{t \in N} \bar{\psi}(t)\right)-\mathrm{E}\left[\sum_{t \in N} \bar{\varphi}(t) \bar{\psi}^{*}(t)\right]+\mathrm{E}\left[\sum_{t \in N} \varphi(t, Z) \psi^{*}(t, Z)\right] \\
& \quad=: A-B+C .
\end{aligned}
$$

By definition of the Bartlett spectrum and (10),

$$
A=\int_{\mathbb{R}^{m}} \hat{\bar{\varphi}}(v) \hat{\bar{\psi}}^{*}(v) \mu_{N}(\mathrm{~d} v) .
$$

By definition of the intensity $\lambda$,

$$
B=\lambda \int_{\mathbb{R}^{m}} \bar{\varphi}(t) \bar{\psi}(t)^{*} \mathrm{~d} t, \quad C=\lambda \int_{\mathbb{R}^{m}} \mathrm{E}\left[\varphi(t, Z) \psi(t, Z)^{*}\right] \mathrm{d} t .
$$

By the Plancherel-Parseval identity,

$$
B=\lambda \int_{\mathbb{R}^{m}} \hat{\bar{\varphi}}(v) \hat{\bar{\psi}}(v)^{*} \mathrm{~d} v=\lambda \int_{\mathbb{R}^{m}} \overline{\hat{\varphi}}(v) \overline{\hat{\psi}}(v)^{*} \mathrm{~d} v=\lambda \int_{\mathbb{R}^{m}} \mathrm{E}[\hat{\varphi}(v, Z)] \mathrm{E}\left[\hat{\psi}(v, Z)^{*}\right] \mathrm{d} v
$$

and

$$
C=\lambda \mathrm{E}\left[\int_{\mathbb{R}^{m}} \hat{\varphi}(v, Z) \hat{\psi}(v, Z)^{*} \mathrm{~d} v\right]
$$

and (11) follows.

We can now compute the power spectrum of a shot noise.

Corollary 1. Consider the above marked point process (with independent and identically distributed marks) and let $h: \mathbb{R}^{m} \times K \rightarrow \mathbb{R}$ satisfy (10) and (9). Define the shot noise $\{X(t)\}_{t \in \mathbb{R}^{m}}$ by

$$
X(t)=\sum_{s \in N} h(t-s, Z(t))
$$

Its Bochner spectral measure is given by the formula

$$
\mu_{X}(\mathrm{~d} v)=|\mathrm{E}[\hat{h}(v, Z)]|^{2} \mu_{N}(\mathrm{~d} v)+\lambda \operatorname{var}(\hat{h}(v, Z)) \mathrm{d} v .
$$

Proof. It suffices to apply the fundamental isometry formula to $\varphi(t, z)=h(u-t, z)$ and $\psi(t, z)=h(v-t, z)$, to obtain

$$
\begin{aligned}
\operatorname{cov}(X(u), X(v))= & \int_{\mathbb{R}^{m}}|\overline{\hat{h}}(v)|^{2} \exp (-2 \mathrm{i} \pi\langle v, u-v\rangle) \mu_{N}(\mathrm{~d} v) \\
& +\lambda \int_{\mathbb{R}^{m}} \operatorname{var}(\hat{h}(v, Z)) \exp (-2 \mathrm{i} \pi\langle v, u-v\rangle) \mathrm{d} \nu .
\end{aligned}
$$




\subsection{Jittered point processes}

If the Bartlett spectrum $\mu_{N}$ of a wide-sense stationary point process $N$ is known, what is the Bartlett spectrum $\mu_{\tilde{N}}$ of the point process obtained by independent and identically distributed displacements of the points of $N$ ? We have the following result.

Corollary 2. Consider the marked point process of Theorem 2 , with $K=\mathbb{R}^{m}$. A point process $\tilde{N}$ is defined by

$$
\tilde{N}=\{t+Z(t), t \in N\} .
$$

Then, if $\lambda$ is the intensity of $N$ and $\mu_{\tilde{N}}$ the Bartlett spectrum of $\tilde{N}$, we have

$$
\mu_{\tilde{N}}(\mathrm{~d} v)=\left|\psi_{Z}(v)\right|^{2} \mu_{N}(\mathrm{~d} v)+\lambda\left(1-\left|\psi_{Z}(v)\right|^{2}\right) \mathrm{d} v
$$

where

$$
\psi_{Z}(v)=\mathrm{E}\left[\mathrm{e}^{2 \mathrm{i} \pi\langle v, Z\rangle}\right]
$$

is the characteristic function of the random displacements distributed according to $Q$. We can take

$$
B_{\tilde{N}}=\left\{\tilde{\varphi}: \tilde{\varphi} \in L_{\mathbb{C}}^{1}\left(\mathbb{R}^{m}\right) \cap L_{\mathbb{C}}^{2}\left(\mathbb{R}^{m}\right), \mathrm{E}[\tilde{\varphi}(t+Z)] \in B_{N}\right\} .
$$

Proof. Define $\varphi(t, z):=\tilde{\varphi}(t+z)$. Conditions (8) and (9) for the function $\varphi$ are respectively equivalent to the conditions $\tilde{\varphi} \in L_{\mathbb{C}}^{1}\left(\mathbb{R}^{m}\right)$ and $\tilde{\varphi} \in L_{\mathbb{C}}^{2}\left(\mathbb{R}^{m}\right)$ since, for any $p \geq 0$,

$$
\mathrm{E}\left[\int_{\mathbb{R}^{m}}|\varphi(t, Z)|^{p} \mathrm{~d} t\right]=\int_{\mathbb{R}^{m}}|\tilde{\varphi}(t)|^{p} \mathrm{~d} t .
$$

Condition (10) for the function $\varphi$ is satisfied by the ad hoc definition of $B_{\tilde{N}}$. We may therefore apply Theorem 2 . We have

$$
\begin{aligned}
\hat{\varphi}(\nu, z) & =\mathrm{e}^{2 \mathrm{i} \pi\langle v, z\rangle} \hat{\tilde{\varphi}}(v), \\
\hat{\bar{\varphi}}(v) & =\overline{\hat{\varphi}}(v)=\psi_{Z}(v) \hat{\tilde{\varphi}}(v), \\
\operatorname{cov}\left(\hat{\varphi}(v, Z), \hat{\varphi}(\nu, Z)^{*}\right) & =\left(1-\left|\psi_{Z}(v)\right|^{2}\right)|\hat{\tilde{\varphi}}(v)|^{2} .
\end{aligned}
$$

Also,

$$
\operatorname{var}\left(\int_{\mathbb{R}^{m}} \tilde{\varphi}(t) \tilde{N}(\mathrm{~d} t)\right)=\operatorname{var}\left(\sum_{t \in N} \tilde{\varphi}(t+Z(t))\right)
$$

and, therefore, by (11),

$$
\operatorname{var}\left(\int_{\mathbb{R}^{m}} \tilde{\varphi}(t) \tilde{N}(\mathrm{~d} t)\right)=\int_{\mathbb{R}^{m}}|\hat{\tilde{\varphi}}(v)|^{2} \mu_{\tilde{N}}(\mathrm{~d} v),
$$

where $\mu_{\tilde{N}}$ is given by (12).

Example 3. (Jittered regular grid.) Consider the case in which $N$ is the grid process of Example 1. We can take

$$
B_{\tilde{N}}=\left\{\tilde{\varphi}: \tilde{\varphi} \in L_{\mathbb{C}}^{1}\left(\mathbb{R}^{2}\right) \cap L_{\mathbb{C}}^{2}\left(\mathbb{R}^{2}\right), \sum_{n_{1}, n_{2} \in \mathbb{Z}}\left|\hat{\tilde{\varphi}}\left(\frac{n_{1}}{T_{1}}, \frac{n_{2}}{T_{2}}\right)\right|<\infty\right\} .
$$

To see this, observe that

$$
|\mathrm{E}[\hat{\tilde{\varphi}}(\cdot+Z)](v)|=|\hat{\tilde{\varphi}}(v)| .
$$


Thus, the condition $\mathrm{E}[\tilde{\varphi}(t+Z)] \in B_{N}$ is equivalent to

$$
\sum_{n_{1}, n_{2} \in \mathbb{Z}}\left|\hat{\tilde{\varphi}}\left(\frac{n_{1}}{T_{1}}, \frac{n_{2}}{T_{2}}\right)\right|<\infty .
$$

Example 4. (Jittered Cox process.) Consider the case in which $N$ is the Cox process of Example 2. We can take

$$
B_{\tilde{N}}=\left\{\tilde{\varphi}: \tilde{\varphi} \in L_{\mathbb{C}}^{1}\left(\mathbb{R}^{m}\right) \cap L_{\mathbb{C}}^{2}\left(\mathbb{R}^{m}\right)\right\}
$$

Indeed, the condition $\mathrm{E}[\tilde{\varphi}(t+Z)] \in B_{N}$, that is, in this particular case,

$$
\mathrm{E}[\tilde{\varphi}(t+Z)] \in L_{\mathbb{C}}^{1}\left(\mathbb{R}^{m}\right) \cap L_{\mathbb{C}}^{2}\left(\mathbb{R}^{m}\right),
$$

is equivalent to $\tilde{\varphi} \in L_{\mathbb{C}}^{1}\left(\mathbb{R}^{m}\right) \cap L_{\mathbb{C}}^{2}\left(\mathbb{R}^{m}\right)$.

\subsection{Cluster point processes}

Let $N$ be a stationary point process on $\mathbb{R}^{m}$ with intensity $\lambda>0$, Bartlett spectral measure $\mu_{N}$, and set of test functions $\mathscr{B}_{N}$. Let $\{Z(t)\}_{t \in \mathbb{R}^{m}}$ be a collection of independent and identically distributed point processes on $\mathbb{R}^{m}$, independent of $N$. Write $Z(t)(C)=Z(t, C)$ and let $Z$ be a point process on $\mathbb{R}^{m}$ with the same distribution as the common distribution of the $Z(t) \mathrm{s}$. Moreover, let

$$
\psi_{Z}(v)=\mathrm{E}\left[\int_{\mathbb{R}^{m}} \mathrm{e}^{2 \mathrm{i} \pi\langle v, t\rangle} Z(\mathrm{~d} t)\right] .
$$

The existence and finiteness of $\psi_{Z}(v)$ are guaranteed under the condition

$$
\mathrm{E}\left[Z\left(\mathbb{R}^{m}\right)\right]<\infty .
$$

In particular, $Z$ is almost surely a finite point process.

We now define two point process on $\mathbb{R}^{m}, \tilde{N}$ and $\hat{N}$, by

$$
\begin{aligned}
& \tilde{N}(C)=N(C)+\sum_{t \in N} Z(t, C-t), \\
& \hat{N}(C)=\sum_{t \in N} Z(t, C-t) .
\end{aligned}
$$

We would like to compute the Bartlett spectra of $\tilde{N}$ and $\hat{N}$. We start with $\tilde{N}$. Formally,

$$
\begin{aligned}
\operatorname{var}\left(\sum_{t \in \tilde{N}} \varphi(t)\right) & =\operatorname{var}\left(\sum_{t \in N}\left\{\varphi(t)+\int_{\mathbb{R}^{m}} \varphi(t+s) Z(t, \mathrm{~d} s)\right\}\right) \\
& =\operatorname{var}\left(\sum_{t \in N} \varphi(t, Z(t))\right),
\end{aligned}
$$

where, if $z$ is a measure and $Z$ is a random measure,

$$
\varphi(t, Z)=\varphi(t)+\int_{\mathbb{R}^{m}} \varphi(t+s) Z(\mathrm{~d} s),
$$


and

$$
\begin{gathered}
\mathrm{E}[\varphi(t, Z)]=\varphi(t)+\mathrm{E}\left[\int_{\mathbb{R}^{m}} \varphi(t+s) Z(\mathrm{~d} s)\right], \\
\hat{\varphi}(v, z)=\hat{\varphi}(v)+\int_{\mathbb{R}^{m}}\left(\int_{\mathbb{R}^{m}} \varphi(t+s) z(\mathrm{~d} s)\right) \mathrm{e}^{-2 \mathrm{i} \pi\langle v, t\rangle} \mathrm{d} t \\
=\hat{\varphi}(v)+\int_{\mathbb{R}^{m}}\left(\int_{\mathbb{R}^{m}} \varphi(t+s) \mathrm{e}^{-2 \mathrm{i} \pi\langle v, t\rangle} \mathrm{d} t\right) z(\mathrm{~d} s) \\
=\hat{\varphi}(v)+\int_{\mathbb{R}^{m}} \hat{\varphi}(v) \mathrm{e}^{2 \mathrm{i} \pi\langle v, s\rangle} z(\mathrm{~d} s) \\
=\hat{\varphi}(v)\left(1+\int_{\mathbb{R}^{m}} \mathrm{e}^{2 \mathrm{i} \pi\langle v, s\rangle} z(\mathrm{~d} s)\right) .
\end{gathered}
$$

Note that exchanging the order of integration is not a problem if $z$ is a finite point process, in particular if $z$ is replaced by its random version $Z$. Also note that $\varphi \in \mathscr{B}_{\tilde{N}}$, where $\mathscr{B}_{\tilde{N}}$ is the set of test functions for $\tilde{N}$, is necessarily in $L_{\mathbb{C}}^{1}\left(\mathbb{R}^{m}\right)$, since $\mathcal{B}_{\tilde{N}} \subseteq L_{\mathbb{C}}^{1}\left(\mathbb{R}^{m}\right)$.

We also have

$$
\hat{\bar{\varphi}}(v)=\hat{\varphi}(v)\left(1+\psi_{Z}(v)\right)
$$

By applying Theorem 2 formally, we obtain

$$
\begin{aligned}
\operatorname{var}\left(\sum_{t \in N} \varphi(t, Z(t))\right)= & \int_{\mathbb{R}^{m}}|\hat{\varphi}(v)|^{2}\left|1+\psi_{Z}(v)\right|^{2} \mu_{N}(\mathrm{~d} v) \\
& +\lambda \int_{\mathbb{R}^{m}}|\hat{\varphi}(v)|^{2} \operatorname{var}\left(1+\int_{\mathbb{R}^{m}} \mathrm{e}^{2 \mathrm{i} \pi\langle v, s\rangle} Z(\mathrm{~d} s)\right) \mathrm{d} \nu
\end{aligned}
$$

Observe that

$$
\operatorname{var}\left(1+\int_{\mathbb{R}^{m}} \mathrm{e}^{2 \mathrm{i} \pi\langle v, s\rangle} Z(\mathrm{~d} s)\right)=\operatorname{var}\left(\int_{\mathbb{R}^{m}} \mathrm{e}^{2 \mathrm{i} \pi\langle v, s\rangle} Z(\mathrm{~d} s)\right) ;
$$

this implies that

$$
\operatorname{var}\left(\sum_{t \in \tilde{N}} \varphi(t)\right)=\int_{\mathbb{R}^{m}}|\hat{\varphi}(v)|^{2} \mu_{\tilde{N}}(\mathrm{~d} v)
$$

where

$$
\mu_{\tilde{N}}(\mathrm{~d} v)=\left|1+\psi_{Z}(v)\right|^{2} \mu_{N}(\mathrm{~d} v)+\lambda \operatorname{var}\left(\int_{\mathbb{R}^{m}} \mathrm{e}^{2 \mathrm{i} \pi\langle v, s\rangle} Z(\mathrm{~d} s)\right) \mathrm{d} \nu
$$

is the Bartlett spectrum of $\tilde{N}$. Similar computations give

$$
\mu_{\hat{N}}(\mathrm{~d} \nu)=\left|\psi_{Z}(v)\right|^{2} \mu_{N}(\mathrm{~d} v)+\lambda \operatorname{var}\left(\int_{\mathbb{R}^{m}} \mathrm{e}^{2 \mathrm{i} \pi\langle v, s\rangle} Z(\mathrm{~d} s)\right) \mathrm{d} \nu
$$

for the Bartlett spectrum of $\hat{N}$.

To obtain the corresponding domains $\mathscr{B}_{\tilde{N}}$ and $\mathscr{B}_{\hat{N}}$, it suffices to require that the conditions on $\varphi(t, z)$ required in Theorem 2 are satisfied. 


\section{Spatial Hawkes processes}

\subsection{Spatial branching point processes}

The Hawkes point process $N$ on $\mathbb{R}^{m}$ is a spatial branching point process. It is constructed as follows. Let $N_{0}$ be a simple, stationary second-order point process with Bartlett spectrum $\mu_{0}$ and set of admissible functions $B_{N_{0}}$. This point process is called the 'ancestor process'. Let $\left\{Z_{n}(t)\right\}_{n \geq 0, t \in \mathbb{R}^{m}}$, be a family of independent and identically distributed random variables, independent of $N_{0}$, with values in the measurable space $(K, \mathcal{K})$ and common distribution $Q$. Let

$$
N=\sum_{n \geq 0} N_{n}
$$

where each $N_{n}$ is the basic point process on $\mathbb{R}^{m}$ of a marked point process $\bar{N}_{n}$ on $\mathbb{R}^{m} \times K$ with independent and identically distributed marks $\left\{Z_{n}(t)\right\}_{t \in \mathbb{R}^{m}}$, that is,

$$
\bar{N}_{n}(C \times L)=\sum_{t \in N_{n}} \mathbf{1}_{C}(t) \mathbf{1}_{L}\left(Z_{n}(t)\right)
$$

where $C \subseteq \mathbb{R}^{m}$ is a Borel set, $L \in \mathcal{K}$, and $\mathbf{1}_{A}$ is the indicator function of the generic set $A$. The sequence of point processes $\left\{N_{n}\right\}_{n \geq 1}$ is constructed recursively as follows. First, let $h: \mathbb{R}^{m} \times K \rightarrow \mathbb{R}$ be a nonnegative rate function such that the quantity

$$
\rho:=\int_{\mathbb{R}^{m}} \mathrm{E}[h(t, Z)] \mathrm{d} t
$$

is finite, where $Z$ is a $K$-valued random variable with distribution $Q$ (the general mark distribution). We denote by $\mathcal{F}_{n}$ the sigma-field recording all the events relative to $\bar{N}_{0}, \ldots, \bar{N}_{n}$. Then, conditionally on $\mathcal{F}_{n-1}, N_{n}$ is a Poisson process on $\mathbb{R}^{m}$ with intensity

$$
\lambda_{n}(t)=\int_{\mathbb{R}^{m}} \int_{K} h(t-s, z) \bar{N}_{n-1}(\mathrm{~d} s \times \mathrm{d} z)=\sum_{s \in N_{n-1}} h\left(t-s, Z_{n-1}(s)\right) .
$$

We refer to $N_{n}$ as the $n$th generation point process, and give it the following interpretation. Each point $a \in N_{n-1}$ of generation $n-1$ creates descendants in the next generation according to a Poisson process of intensity $h\left(t-a, Z_{n-1}(a)\right.$ ). For each ancestor (each point $a \in N_{0}$ ), we therefore have $\rho$ direct descendants, on average. We write

$$
N^{\prime}=\sum_{n \geq 1} N_{n}, \quad \bar{N}^{\prime}=\sum_{n \geq 1} \bar{N}_{n} .
$$

From (13) and Campbell's theorem, we see that

$$
\lambda_{n}=\lambda_{n-1} \int_{\mathbb{R}^{m}} \mathrm{E}[h(t, Z)] \mathrm{d} t,
$$

where $\lambda_{n}=\mathrm{E}\left[\lambda_{n}(t)\right]$, and, therefore, that the average intensity $\lambda^{\prime}$ of $N^{\prime}$ obeys

$$
\lambda^{\prime}=\rho \lambda_{0}+\rho \lambda^{\prime}
$$

Thus, if $\lambda_{0}>0$, in order for $N^{\prime}$ to have a finite intensity it is necessary that

$$
\rho<1
$$


In this case, each ancestor is the root of an eventually extinguished branching process, because its average number of progeny is strictly less than 1. Condition (14) will be assumed to hold throughout.

Lemma 1. For $\varphi \in L_{\mathbb{C}}^{1}(\ell \times Q) \cap L_{\mathbb{C}}^{2}(\ell \times Q)$, we have

$$
\operatorname{var}\left(\int_{\mathbb{R}^{m}} \int_{K} \varphi(t, z) \bar{M}^{\prime}(\mathrm{d} t \times \mathrm{d} z)\right)=\lambda^{\prime} \int_{\mathbb{R}^{m}} \mathrm{E}\left[|\varphi(t, Z)|^{2}\right] \mathrm{d} t,
$$

where

$$
\begin{gathered}
\bar{M}^{\prime}(\mathrm{d} t \times \mathrm{d} z)=\bar{N}^{\prime}(\mathrm{d} t \times \mathrm{d} z)-\lambda^{\prime}(t) \mathrm{d} t Q(\mathrm{~d} z), \\
\lambda^{\prime}(t)=\int_{\mathbb{R}^{m}} \int_{K} h\left(t-s, z^{\prime}\right) \bar{N}_{0}\left(\mathrm{~d} t \times \mathrm{d} z^{\prime}\right)+\int_{\mathbb{R}^{m}} \int_{K} h\left(t-s, z^{\prime}\right) \bar{N}^{\prime}\left(\mathrm{d} t \times \mathrm{d} z^{\prime}\right) .
\end{gathered}
$$

Proof. We shall use a simplified notation of the kind

$$
\int_{\mathbb{R}^{m}} \int_{K} \varphi(t, z) \bar{M}^{\prime}(\mathrm{d} t \times \mathrm{d} z)=\int \varphi \mathrm{d} \bar{M}^{\prime} .
$$

We then have

$$
\int \varphi \mathrm{d} \bar{M}^{\prime}=\sum_{n \geq 1} \int \varphi \mathrm{d} \bar{M}_{n}
$$

where $\bar{M}_{n}(\mathrm{~d} t \times \mathrm{d} z)=\bar{N}_{n}(\mathrm{~d} t \times \mathrm{d} z)-\lambda_{n}(t) \mathrm{d} t Q(\mathrm{~d} z)$. Given $\mathcal{F}_{n-1}, \bar{N}_{n}$ is a Poisson process with mean measure $\lambda_{n}(t) Q(\mathrm{~d} z) \mathrm{d} t$ and, therefore, by standard properties of Poisson processes, we have

$$
\operatorname{var}\left(\int \varphi \mathrm{d} \bar{M}_{n} \mid \mathcal{F}_{n-1}\right)=\int_{\mathbb{R}^{m}} \mathrm{E}\left[\varphi^{2}(t, Z)\right] \lambda_{n}(t) \mathrm{d} t,
$$

and

$$
\mathrm{E}\left[\int \varphi \mathrm{d} \bar{M}_{n} \mid \mathcal{F}_{n-1}\right]=0 .
$$

Therefore, by the conditional variance formula,

$$
\begin{aligned}
\operatorname{var}\left(\int \varphi \mathrm{d} \bar{M}_{n}\right) & =\mathrm{E}\left[\operatorname{var}\left(\int \varphi \mathrm{d} \bar{M}_{n} \mid \mathcal{F}_{n-1}\right)\right]+\operatorname{var}\left(\mathrm{E}\left[\int \varphi \mathrm{d} \bar{M}_{n} \mid \mathcal{F}_{n-1}\right]\right) \\
& =\lambda_{n} \int_{\mathbb{R}^{m}} \mathrm{E}\left[\varphi^{2}(t, Z)\right] \mathrm{d} t .
\end{aligned}
$$

Also, for $j, k \geq 1$,

$$
\mathrm{E}\left[\left(\int \varphi \mathrm{d} \bar{M}_{j}\right)\left(\int \varphi \mathrm{d} \bar{M}_{j+k}\right)\right]=\mathrm{E}\left[\left(\int \varphi \mathrm{d} \bar{M}_{j}\right) \mathrm{E}\left[\int \varphi \mathrm{d} \bar{M}_{j+k} \mid \mathcal{F}_{j+k-1}\right]\right]=0 .
$$

Therefore,

$$
\begin{aligned}
\operatorname{var}\left(\int \varphi \mathrm{d} \bar{M}^{\prime}\right) & =\sum_{n \geq 1} \operatorname{var}\left(\int \varphi \mathrm{d} \bar{M}_{n}\right) \\
& =\left(\sum_{n \geq 1} \lambda_{n}\right) \int_{\mathbb{R}^{m}} \mathrm{E}\left[\varphi^{2}(t, Z)\right] \mathrm{d} t \\
& =\lambda^{\prime} \int_{\mathbb{R}^{m}} \mathrm{E}\left[\varphi^{2}(t, Z)\right] \mathrm{d} t .
\end{aligned}
$$




\subsection{Spectrum of the Hawkes process}

Lemma 2. (a) Suppose that

$$
\mathrm{E}\left[\left(\int_{\mathbb{R}^{m}} h(t, Z) \mathrm{d} t\right)^{2}\right]<\infty
$$

For any given $F \in L_{\mathbb{C}}^{1}(\ell \times Q) \cap L_{\mathbb{C}}^{2}(\ell \times Q)$, there exists a unique $\varphi \in L_{\mathbb{C}}^{1}(\ell \times Q) \cap L_{\mathbb{C}}^{2}(\ell \times Q)$ such that

$$
\varphi(t, z)-\int_{\mathbb{R}^{m}} h(s-t, z) \mathrm{E}[\varphi(s, Z)] \mathrm{d} s=F(t, z) .
$$

(b) For a given $f \in L_{\mathbb{C}}^{1}\left(\mathbb{R}^{m}\right) \cap L_{\mathbb{C}}^{2}\left(\mathbb{R}^{m}\right)$, there exists a unique $\varphi \in L_{\mathbb{C}}^{1}(\ell \times Q) \cap L_{\mathbb{C}}^{2}(\ell \times Q)$ such that

$$
\varphi(t, z)-\int_{\mathbb{R}^{m}} h(s-t, z) \mathrm{E}[\varphi(s, Z)] \mathrm{d} s=f(t) .
$$

Proof. (a) For a function $v(t, z)$, denote $\mathrm{E}[v(t, Z)]$ by $\bar{v}(t)$ and $v(-t, z)$ by $\check{v}(t, z)$. Note that $F \in L_{\mathbb{C}}^{1}(\ell \times Q) \cap L_{\mathbb{C}}^{2}(\ell \times Q)$ implies $\bar{F} \in L_{\mathbb{C}}^{1}\left(\mathbb{R}^{m}\right) \cap L_{\mathbb{C}}^{2}\left(\mathbb{R}^{m}\right)$. Let $h$ and $F$ be as in the statement of the lemma, and consider the renewal equation

$$
g=\bar{F}+\check{\bar{h}} * g \text {. }
$$

Since $\bar{F} \in L_{\mathbb{C}}^{1}\left(\mathbb{R}^{m}\right) \cap L_{\mathbb{C}}^{2}\left(\mathbb{R}^{m}\right)$ and (14) holds, there exists a unique solution $g \in L_{\mathbb{C}}^{1}\left(\mathbb{R}^{m}\right) \cap$ $L_{\mathbb{C}}^{2}\left(\mathbb{R}^{m}\right)$ given by

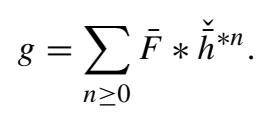

(The convergence of this series in $L_{\mathbb{C}}^{1}\left(\mathbb{R}^{m}\right)$ as well as in $L_{\mathbb{C}}^{2}\left(\mathbb{R}^{m}\right)$ is guaranteed by the inequalities $\|a * b\|_{L^{1}} \leq_{\check{\Sigma}}\|a\|_{L^{1}}\|b\|_{L^{1}}$ and $\|a * b\|_{L^{2}} \leq\|a\|_{L^{1}}\|b\|_{L^{2}}$; uniqueness follows from the equality $g-g^{\prime}=\bar{h} *\left(g-g^{\prime}\right)$, where $g^{\prime}$ is another candidate solution, which implies that $\left\|g-g^{\prime}\right\|_{L^{1}} \leq\|\bar{h}\|_{L^{1}}\left\|g-g^{\prime}\right\|_{L^{1}}$ and, hence, by (14), that necessarily $\left\|g-g^{\prime}\right\|_{L^{1}}=0$.) The Fourier transform of $g$ is

$$
\hat{g}(v)=\frac{\mathrm{E}[\hat{F}(\nu, Z)]}{1-\mathrm{E}\left[\hat{h}(v, Z)^{*}\right]} .
$$

Now define $\varphi(t, z)$ by

$$
\varphi(t, z)=\int_{\mathbb{R}^{m}} h(s-t, z) g(s) \mathrm{d} s+F(t, z) .
$$

We have

$$
\mathrm{E}\left[\int_{\mathbb{R}^{m}}|\varphi(t, Z)| \mathrm{d} t\right] \leq \mathrm{E}\left[\int_{\mathbb{R}^{m}}|F(t, Z)| \mathrm{d} t\right]+\mathrm{E}\left[\int_{\mathbb{R}^{m}}|h(t, Z)| \mathrm{d} t\right] \int_{\mathbb{R}^{m}}|g(t)| \mathrm{d} t<\infty,
$$

because $g \in L_{\mathbb{C}}^{1}\left(\mathbb{R}^{m}\right)$ and $F, h \in L_{\mathbb{C}}^{1}(\ell \times Q)$. Therefore, $\varphi \in L_{\mathbb{C}}^{1}(\ell \times Q)$. We now show that $\varphi \in L_{\mathbb{C}}^{2}(\ell \times Q)$. It suffices to show that $\hat{\varphi}(v, z) \in L_{\mathbb{C}}^{2}(\ell \times Q)$ because then, by using the Plancherel-Parseval identity, we have

$$
\mathrm{E}\left[\int_{\mathbb{R}^{m}}|\varphi(t, Z)|^{2} \mathrm{~d} t\right]=\mathrm{E}\left[\int_{\mathbb{R}^{m}}|\hat{\varphi}(v, Z)|^{2} \mathrm{~d} v\right]<\infty .
$$


For this purpose, we take the Fourier transform of (19) for fixed $z$, i.e.

$$
\hat{\varphi}(v, z)=\hat{h}(v, z)^{*} \hat{g}(v)+\hat{F}(v, z),
$$

or (for future reference), in view of (18),

$$
\hat{\varphi}(\nu, z)=\hat{F}(v, z)+\frac{\hat{h}(v, z)^{*} \mathrm{E}[\hat{F}(v, Z)]}{1-\mathrm{E}\left[\hat{h}(v, Z)^{*}\right]} .
$$

We will show that $\hat{\varphi}(\nu, z) \in L_{\mathbb{C}}^{2}(\ell \times Q)$. Since $F(t, z) \in L_{\mathbb{C}}^{2}(\ell \times Q)$, it follows from the Plancherel-Parseval identity that $\hat{F}(v, z) \in L_{\mathbb{C}}^{2}(\ell \times Q)$. It remains to show that

$$
\hat{h}(\nu, z) \hat{g}(\nu) \in L_{\mathbb{C}}^{2}(\ell \times Q) ;
$$

this follows from the facts that $\hat{g} \in L_{\mathbb{C}}^{2}\left(\mathbb{R}^{m}\right)$ and

$$
\mathrm{E}\left[|\hat{h}(v, Z)|^{2}\right]=\mathrm{E}\left[\left|\int_{\mathbb{R}^{m}} h(t, Z) \mathrm{e}^{2 \mathrm{i} \pi v t} \mathrm{~d} t\right|^{2}\right] \leq \mathrm{E}\left[\left|\int_{\mathbb{R}^{m}} h(t, Z) \mathrm{d} t\right|^{2}\right]
$$

is a finite constant (by (15)) independent of $\nu$.

(b) This is clearly a particular case of (a). For future reference, we note that in this case the following relation holds:

$$
\hat{\varphi}(v, z)=\hat{f}(v)\left(1+\frac{\hat{h}^{*}(v, z)}{1-\mathrm{E}\left[\hat{h}^{*}(v, Z)\right]}\right) .
$$

Theorem 3. Let h $(t, z)$ satisfy (14) and (15). The Bartlett spectrum of the Hawkes point process $N$ defined above is

$$
\mu_{N}(\mathrm{~d} v)=\frac{1}{|1-\mathrm{E}[\hat{h}(v, Z)]|^{2}}\left[\mu_{0}(\mathrm{~d} v)+\lambda^{\prime} \mathrm{d} v+\lambda \operatorname{var}(\hat{h}(v, Z) \mathrm{d} v)\right],
$$

where $\lambda=\lambda_{0} /(1-\rho)$. For $B_{N}$, we can take the set of functions $f \in L_{\mathbb{C}}^{1}\left(\mathbb{R}^{m}\right) \cap L_{\mathbb{C}}^{2}\left(\mathbb{R}^{m}\right)$ such that the solution to (17) satisfies $\mathrm{E}[\varphi(\cdot, Z)] \in B_{N_{0}}$.

Proof. Let $\varphi$ be the solution to (17). Then

$$
\begin{aligned}
\int_{\mathbb{R}^{m}} \int_{K} \varphi(t, z) \bar{M}^{\prime}(\mathrm{d} t \times \mathrm{d} z) & =\int_{\mathbb{R}^{m}} \int_{K} \varphi(t, z)\left(\bar{N}^{\prime}(\mathrm{d} t \times \mathrm{d} z)-\lambda^{\prime}(t) Q(\mathrm{~d} z) \mathrm{d} t\right) \\
& =\int_{\mathbb{R}^{m}} \int_{K} \varphi(t, z) \bar{N}^{\prime}(\mathrm{d} t \times \mathrm{d} z)-\int_{\mathbb{R}^{m}} \int_{K} \varphi(t, z) \lambda^{\prime}(t) Q(\mathrm{~d} z) \mathrm{d} t
\end{aligned}
$$

and

$$
\begin{aligned}
\int_{\mathbb{R}^{m}} \int_{K} \varphi(t, z) \lambda^{\prime}(t) Q(\mathrm{~d} z) \mathrm{d} t & =\int_{\mathbb{R}^{m}} \int_{K} \varphi(t, z)\left(\int_{\mathbb{R}^{m}} \int_{K} h\left(t-s, z^{\prime}\right) \bar{N}\left(\mathrm{~d} s \times \mathrm{d} z^{\prime}\right)\right) Q(\mathrm{~d} z) \mathrm{d} t \\
& =\int_{\mathbb{R}^{m} \times \mathbb{R}^{m} \times K} h\left(t-s, z^{\prime}\right) \mathrm{E}[\varphi(t, Z)] \mathrm{d} t \bar{N}\left(\mathrm{~d} s \times \mathrm{d} z^{\prime}\right) \\
& =\int_{\mathbb{R}^{m}} \int_{K}\left(\check{h}\left(s-\cdot, z^{\prime}\right) * \mathrm{E}[\varphi(\cdot, Z)]\right)(s) \bar{N}\left(\mathrm{~d} s \times \mathrm{d} z^{\prime}\right) .
\end{aligned}
$$


Therefore, since $\bar{N}=\bar{N}^{\prime}+\bar{N}_{0}$, we have

$$
\begin{aligned}
\int_{\mathbb{R}^{m}} \int_{K} \varphi(t, z) \bar{M}^{\prime}(\mathrm{d} t \times \mathrm{d} z)= & \int_{\mathbb{R}^{m}} \int_{K}(\varphi(t, z)-(\check{h}(t-\cdot, z) * \mathrm{E}[\varphi(\cdot, Z)])(t)) \bar{N}(\mathrm{~d} t \times \mathrm{d} z) \\
& -\int_{\mathbb{R}^{m}} \int_{K} \varphi(t, z) \bar{N}_{0}(\mathrm{~d} t \times \mathrm{d} z) .
\end{aligned}
$$

Take an $f \in B_{N}$ (in particular, an $f \in L_{\mathbb{C}}^{1}\left(\mathbb{R}^{m}\right) \cap L_{\mathbb{C}}^{2}\left(\mathbb{R}^{m}\right)$ ). We then have

$$
\int_{\mathbb{R}^{m}} \int_{K} \varphi(t, z) \bar{M}^{\prime}(\mathrm{d} t \times \mathrm{d} z)+\int_{\mathbb{R}^{m}} \int_{K} \varphi(t, z) \bar{N}_{0}(\mathrm{~d} t \times \mathrm{d} z)=\int_{\mathbb{R}^{m}} f(t) N(\mathrm{~d} t) .
$$

Also, by the isometry lemma,

$$
\operatorname{var}\left(\int_{\mathbb{R}^{m}} \int_{K} \varphi(t, z) \bar{M}^{\prime}(\mathrm{d} t \times \mathrm{d} z)\right)=\lambda^{\prime} \int_{\mathbb{R}^{m}} \mathrm{E}\left[|\varphi(t, Z)|^{2}\right] \mathrm{d} t=\lambda^{\prime} \int_{\mathbb{R}^{m}} \mathrm{E}\left[|\hat{\varphi}(\nu, Z)|^{2}\right] \mathrm{d} \nu .
$$

Now,

$$
\begin{aligned}
& \mathrm{E}\left[\int_{\mathbb{R}^{m}} \int_{K} \varphi(t, z) \bar{M}^{\prime}(\mathrm{d} t \times \mathrm{d} z) \int_{\mathbb{R}^{m}} \int_{K} \varphi(t, z) \bar{N}_{0}(\mathrm{~d} t \times \mathrm{d} z)\right] \\
& \quad=\mathrm{E}\left[\mathrm{E}\left[\int_{\mathbb{R}^{m}} \int_{K} \varphi(t, z) \bar{M}^{\prime}(\mathrm{d} t \times \mathrm{d} z) \mid \mathcal{F}_{0}\right] \int_{\mathbb{R}^{m}} \int_{K} \varphi(t, z) \bar{N}_{0}(\mathrm{~d} t \times \mathrm{d} z)\right]=0
\end{aligned}
$$

and, therefore,

$$
\begin{aligned}
& \operatorname{var}\left(\int_{\mathbb{R}^{m}} \int_{K} \varphi(t, z) \bar{M}^{\prime}(\mathrm{d} t \times \mathrm{d} z)+\int_{\mathbb{R}^{m}} \int_{K} \varphi(t, z) \bar{N}_{0}(\mathrm{~d} t \times \mathrm{d} z)\right) \\
& \quad=\operatorname{var}\left(\int_{\mathbb{R}^{m}} \int_{K} \varphi(t, z) \bar{M}^{\prime}(\mathrm{d} t \times \mathrm{d} z)\right)+\operatorname{var}\left(\int_{\mathbb{R}^{m}} \int_{K} \varphi(t, z) \bar{N}_{0}(\mathrm{~d} t \times \mathrm{d} z)\right) \\
& \quad=\lambda^{\prime} \int_{\mathbb{R}^{m}} \mathrm{E}\left[|\varphi(t, Z)|^{2}\right] \mathrm{d} t+\operatorname{var}\left(\int_{\mathbb{R}^{m}} \int_{K} \varphi(t, z) \bar{N}_{0}(\mathrm{~d} t \times \mathrm{d} z)\right) .
\end{aligned}
$$

Furthermore,

$$
\operatorname{var}\left(\int_{\mathbb{R}^{m}} \int_{K} \varphi(t, z) \bar{N}_{0}(\mathrm{~d} t \times \mathrm{d} z)\right)=\int_{\mathbb{R}^{m}}|\mathrm{E}[\hat{\varphi}(\nu, Z)]|^{2} \mu_{0}(\mathrm{~d} \nu)+\lambda_{0} \int_{\mathbb{R}^{m}} \operatorname{var}(\hat{\varphi}(\nu, Z)) \mathrm{d} \nu .
$$

Combining the above, we have

$$
\begin{aligned}
\operatorname{var}\left(\int_{\mathbb{R}^{m}} f(t) N(\mathrm{~d} t)\right)= & \lambda^{\prime} \int_{\mathbb{R}^{m}} \mathrm{E}\left[|\hat{\varphi}(\nu, Z)|^{2}\right] \mathrm{d} \nu+\int_{\mathbb{R}^{m}}|\mathrm{E}[\hat{\varphi}(\nu, Z)]|^{2} \mu_{0}(\mathrm{~d} v) \\
& +\lambda_{0} \int_{\mathbb{R}^{m}} \operatorname{var}(\hat{\varphi}(v, Z)) \mathrm{d} v \\
= & : A+B+C
\end{aligned}
$$

By (20),

$$
\begin{aligned}
& A=\lambda^{\prime} \int_{\mathbb{R}^{m}}|\hat{f}(v)|^{2} \frac{1+\operatorname{var}(\hat{h}(\nu, Z))}{|1-\mathrm{E}[\hat{h}(v, Z)]|^{2}} \mathrm{~d} \nu, \\
& B=\int_{\mathbb{R}^{m}}|\hat{f}(v)|^{2} \frac{1}{|1-\mathrm{E}[\hat{h}(v, Z)]|^{2}} \mu_{0}(\mathrm{~d} v), \\
& C=\int_{\mathbb{R}^{m}}|\hat{f}(v)|^{2} \lambda_{0} \frac{\operatorname{var}(\hat{h}(\nu, Z))}{|1-\mathrm{E}[\hat{h}(v, Z)]|^{2}} \mathrm{~d} \nu .
\end{aligned}
$$


Recalling that $\lambda^{\prime}=\rho \lambda_{0} /(1-\rho)$, we finally obtain

$$
\begin{aligned}
& \operatorname{var}\left(\int_{\mathbb{R}^{m}} f(t) N(\mathrm{~d} t)\right) \\
& \quad=\int_{\mathbb{R}^{m}}|\hat{f}(v)|^{2}\left(\frac{1}{|1-\mathrm{E}[\hat{h}(\nu, Z)]|^{2}}\right)\left(\mu_{0}(\mathrm{~d} \nu)+\lambda^{\prime} \mathrm{d} \nu+\lambda \operatorname{var}(\hat{h}(\nu, Z)) \mathrm{d} \nu\right)
\end{aligned}
$$

for all $f \in L_{\mathbb{C}}^{1}\left(\mathbb{R}^{m}\right) \cap L_{\mathbb{C}}^{2}\left(\mathbb{R}^{m}\right)$, which allows us to identify $\mu_{N}$ as the expression given in (21).

Example 5. (Coxian ancestor process.) In the particular case in which $N_{0}$ is a Cox process as in Example 2, we may take $B_{N}=L_{\mathbb{C}}^{1}\left(\mathbb{R}^{m}\right) \cap L_{\mathbb{C}}^{2}\left(\mathbb{R}^{m}\right)$.

Example 6. (The original Hawkes process.) In the particular case in which

$$
h(t, Z)=h(t)
$$

we have $\hat{h}(v, Z)=\hat{h}(v)$ and

$$
\mu_{N}(\mathrm{~d} v)=\frac{1}{|1-\hat{h}(\nu)|^{2}}\left[\mu_{0}(\mathrm{~d} \nu)+\lambda^{\prime} \mathrm{d} \nu\right] .
$$

If, in addition, $N_{0}$ is a Poisson process with average intensity $\alpha$, since $\alpha+\lambda^{\prime}=\lambda$ we recover the original formula of Hawkes:

$$
\mu_{N}(\mathrm{~d} v)=\frac{\lambda \mathrm{d} v}{|1-\hat{h}(v)|^{2}} .
$$

\subsection{Spatial linear birth-death processes}

We consider a shot noise, based on the Hawkes branching point process $N$ of the previous section, defined by

$$
X(t)=\sum_{s \in N} \alpha(t-s, Z(s)) .
$$

Note that its spectral characteristics cannot be derived from Theorem 2, since now the marks $Z(s)$ and the process $N$ are not mutually independent.

Example 7. In the univariate case, i.e. $\mathbb{R}^{m}=\mathbb{R}$, we write

$$
X(t)=\sum_{n \in \mathbb{Z}} \alpha\left(t-T_{n}, Z_{n}\right) .
$$

To further specialize this example, take

$$
h(t, z)=\beta \mathbf{1}_{[0, z]}(t), \quad \alpha(t, z)=\mathbf{1}_{[0, z]}(t) .
$$

Therefore, interpreting $T_{n}$ as the birth time of individual $n$ in the colony and $Z_{n}$ as its lifetime gives

$$
X(t)=\sum_{n \in \mathbb{Z}} \mathbf{1}_{(-\infty, t]}\left(T_{n}\right) \mathbf{1}_{(t, \infty)}\left(T_{n}+Z_{n}\right)
$$

for the number of individuals in the colony. If, moreover, we assume that $Z_{n}$ is exponentially distributed with parameter $\gamma$ and that the process $N_{0}$ of ancestors is Poisson with intensity $\lambda_{0}$, then the process $\{X(t)\}$ is a Markov birth-death process with infinitesimal generator $Q=\left(q_{i j}\right)$ given by $q_{i, i+1}=\lambda_{0}+\beta i$ and $q_{i, i-1}=\gamma i$, all other terms being 0 . 
Theorem 4. Consider the process $\{X(t)\}$ defined by (23), where $\alpha \in L_{\mathbb{C}}^{1}(\ell \times Q) \cap L_{\mathbb{C}}^{2}(\ell \times Q)$, and let $N$ satisfy the conditions required in Theorem 3. Suppose, moreover, that the solution $\varphi$ to (16) with

$$
F(s, z)=\int_{\mathbb{R}^{m}} \alpha(t-s, z) f(t) \mathrm{d} t
$$

is such that $\mathrm{E}[\varphi(\cdot, Z)] \in B_{N_{0}}$ for any $f \in L_{\mathbb{C}}^{1}\left(\mathbb{R}^{m}\right)$. The Bochner spectral measure $\mu_{X}$ is then given by the expression

$$
\begin{aligned}
|1-\mathrm{E}[\hat{h}(v, Z)]|^{2} \mu_{X}(\mathrm{~d} v)= & |\mathrm{E}[\hat{\alpha}(v, Z)]|^{2}\left(\mu_{0}(\mathrm{~d} v)+\frac{\rho \lambda_{0}}{1-\rho} \mathrm{d} v\right) \\
& +\frac{\lambda_{0}}{1-\rho} \operatorname{var}(\hat{\alpha}(v, Z)(1-\mathrm{E}[\hat{h}(v, Z)])+\hat{h}(v, Z) \mathrm{E}[\hat{\alpha}(v, Z)]) \mathrm{d} v
\end{aligned}
$$

Proof. We seek a measure $\mu_{X}$ such that, for all $f \in L_{\mathbb{C}}^{1}\left(\mathbb{R}^{m}\right)$,

$$
\operatorname{var}\left(\int_{\mathbb{R}^{m}} f(t) X(t) \mathrm{d} t\right)=\int_{\mathbb{R}^{m}}|\hat{f}(v)|^{2} \mu_{X}(\mathrm{~d} v) .
$$

However,

$$
\begin{aligned}
\int_{\mathbb{R}^{m}} f(t) X(t) \mathrm{d} t & =\int_{\mathbb{R}^{m}} f(t)\left(\int_{\mathbb{R}^{m}} \int_{K} \alpha(t-s, z) \bar{N}(\mathrm{~d} s \times \mathrm{d} z)\right) \mathrm{d} t \\
& =\int_{\mathbb{R}^{m}} \int_{K} F(s, z) \bar{N}(\mathrm{~d} s \times \mathrm{d} z),
\end{aligned}
$$

where

$$
F(s, z)=\int_{\mathbb{R}^{m}} \alpha(t-s, z) f(t) \mathrm{d} t=(\check{\alpha}(\cdot, z) * f)(s)
$$

is a function in

$$
L_{\mathbb{C}}^{1}(\ell \times Q) \cap L_{\mathbb{C}}^{2}(\ell \times Q)
$$

(since $\alpha \in L_{\mathbb{C}}^{1}(\ell \times Q) \cap L_{\mathbb{C}}^{2}(\ell \times Q)$ and $\left.f \in L_{\mathbb{C}}^{1}\left(\mathbb{R}^{m}\right)\right)$. We therefore seek a $\mu_{X}$ such that

$$
\operatorname{var}\left(\int_{\mathbb{R}^{m}} \int_{K} F(s, z) \bar{N}(\mathrm{~d} s \times \mathrm{d} z)\right)=\int_{\mathbb{R}^{m}}|\hat{f}(v)|^{2} \mu_{X}(\mathrm{~d} v) .
$$

Following the same calculations as in the proof of Theorem 3, up to (22), and letting $\varphi$ be the unique solution to (16) in $L_{\mathbb{C}}^{1}(\ell \times Q) \cap L_{\mathbb{C}}^{2}(\ell \times Q)$, we have

$$
\int_{\mathbb{R}^{m}} \int_{K} F(s, z) \bar{N}(\mathrm{~d} s \times \mathrm{d} z)=\int_{\mathbb{R}^{m}} \int_{K} \varphi(t, z) \bar{M}^{\prime}(\mathrm{d} t \times \mathrm{d} z)+\int_{\mathbb{R}^{m}} \int_{K} \varphi(t, z) \bar{N}_{0}(\mathrm{~d} t \times \mathrm{d} z) .
$$

Resuming the proof of Theorem 3 after the displayed equation following (22), we obtain

$$
\begin{aligned}
\operatorname{var}\left(\int_{\mathbb{R}^{m}} f(t) X(t) \mathrm{d} t\right)= & \lambda^{\prime} \int_{\mathbb{R}^{m}} \mathrm{E}\left[|\hat{\varphi}(\nu, Z)|^{2}\right] \mathrm{d} \nu+\int_{\mathbb{R}^{m}}|\mathrm{E}[\hat{\varphi}(\nu, Z)]|^{2} \mu_{0}(\mathrm{~d} v) \\
& +\lambda_{0} \int_{\mathbb{R}^{m}} \operatorname{var}(\hat{\varphi}(\nu, Z)) \mathrm{d} v \\
= & : A+B+C,
\end{aligned}
$$


where, using

$$
\begin{aligned}
\hat{\varphi}(v, z) & =\hat{F}(v, z)+\frac{\hat{h}(v, z)^{*} \mathrm{E}[\hat{F}(v, Z)]}{1-\mathrm{E}\left[\hat{h}(v, Z)^{*}\right]} \\
& =\hat{f}(v)\left[\hat{\alpha}(v, z)^{*}+\frac{\hat{h}(v, z)^{*} \mathrm{E}\left[\hat{\alpha}(v, Z)^{*}\right]}{1-\mathrm{E}\left[\hat{h}(v, Z)^{*}\right]}\right],
\end{aligned}
$$

we find that

$$
\begin{aligned}
A & =\lambda^{\prime} \int_{\mathbb{R}^{m}}|\hat{f}(v)|^{2} \frac{\mathrm{E}\left[|\hat{\alpha}(\nu, Z)(1-\mathrm{E}[\hat{h}(v, Z)])+\hat{h}(v, Z) \mathrm{E}[\hat{\alpha}(v, Z)]|^{2}\right]}{|1-\mathrm{E}[\hat{h}(\nu, Z)]|^{2}} \mathrm{~d} \nu, \\
B & =\int_{\mathbb{R}^{m}}|\hat{f}(v)|^{2} \frac{|\mathrm{E}[\hat{\alpha}(\nu, Z)]|^{2}}{|1-\mathrm{E}[\hat{h}(v, Z)]|^{2}} \mu_{0}(\mathrm{~d} v), \\
C & =\lambda_{0} \int_{\mathbb{R}^{m}}|\hat{f}(v)|^{2} \frac{\operatorname{var}(\hat{\alpha}(v, Z)(1-\mathrm{E}[\hat{h}(v, Z)])+\hat{h}(v, Z) \mathrm{E}[\hat{\alpha}(v, Z)])}{|1-\mathrm{E}[\hat{h}(v, Z)]|^{2}} \mathrm{~d} v .
\end{aligned}
$$

Therefore, by using the expression $\lambda^{\prime}=\rho \lambda_{0} /(1-\rho)$, after some algebra we recover (25) with $\mu_{X}(\mathrm{~d} v)$ given by $(24)$.

Example 8. Consider the setup of the previous example, in which $\{X(t)\}$ is a Markov birthdeath process with non-null transition rates $q_{i, i+1}=\lambda_{0}+\beta i$ and $q_{i, i-1}=\gamma i$. We then have the following identifications:

$$
\rho=\frac{\beta}{\gamma}, \quad \mu_{0}(\mathrm{~d} \nu)=\lambda_{0} \mathrm{~d} \nu, \quad \hat{\alpha}(\nu, z)=\frac{1-\mathrm{e}^{-2 \mathrm{i} \pi \nu z}}{2 \mathrm{i} \pi \nu}, \quad \hat{h}(\nu, z)=\beta \frac{1-\mathrm{e}^{-2 \mathrm{i} \pi \nu z}}{2 \mathrm{i} \pi \nu} .
$$

From this we obtain

$$
\begin{gathered}
\mathrm{E}[\hat{\alpha}(v, Z)]=\frac{1}{\gamma+2 \mathrm{i} \pi v}, \\
\hat{\alpha}(v, Z)(1-\mathrm{E}[\hat{h}(v, Z)])+\hat{h}(v, Z) \mathrm{E}[\hat{\alpha}(v, Z)]=\hat{\alpha}(v, Z), \\
\operatorname{var}((1-\mathrm{E}[\hat{h}(v, Z)])+\hat{h}(v, Z) \mathrm{E}[\hat{\alpha}(v, Z)])=\frac{1}{\gamma^{2}+4 \pi^{2} v^{2}} .
\end{gathered}
$$

Combined with (24), these yield the following formula:

$$
\mu_{X}(\mathrm{~d} v)=\frac{2 \lambda_{0}}{\left(\gamma^{2}+4 \pi^{2} v^{2}\right)(1-\beta / \gamma)}
$$

\section{Modulated spike fields}

\subsection{Random sampling}

Random sampling of a continuous-time random signal $\{X(t)\}_{t \in \mathbb{R}}$ yields a sequence of samples

$$
\left\{X\left(T_{n}\right)\right\}_{n \in \mathbb{Z}},
$$

where $\left\{T_{n}\right\}_{n \in \mathbb{Z}}$ is the sequence of points (times of events) of a point process. At the extremities of the spectrum of randomness, we find completely random sampling, or Poisson sampling, where $\left\{T_{n}\right\}_{n \in \mathbb{Z}}$ is a homogeneous Poisson process, and regular sampling, where $T_{n}=n T$ with $T>0$. 
The signal $\{X(t)\}_{t \in \mathbb{R}}$ is called the sampled signal, the point process $\left\{T_{n}\right\}_{n \in \mathbb{Z}}$ the sampler, the sequence (26) the sample sequence, and the process

$$
Y(t)=\sum_{n \in \mathbb{Z}} X\left(T_{n}\right) \delta\left(t-T_{n}\right)
$$

where $\delta(t)$ is the Dirac pseudofunction, the sample comb.

The sampled signal and the sampler are assumed to be independent and stationary (or at least wide-sense stationary for the sampled process). However, we shall also consider dependent sampling. The average intensity $\lambda$ of the sampler is, by definition, the average number of samples per unit time, and the sampling frequency is then $v_{\mathrm{s}}=\lambda$. Two well-known results concern regular sampling and Poisson sampling, the two extremal cases.

Example 9. In regular sampling, the Fourier spectrum of the sample comb is an aliased version of that of the sampled signal. For instance, in the case of a power spectral density,

$$
f_{Y}(v)=\sum_{n \in \mathbb{Z}} f_{X}\left(v-\frac{n}{T}\right)
$$

(where $f_{X}$ and $f_{Y}$ are the power spectral densities of $\{X(t)\}_{t \in \mathbb{R}}$ and $\{Y(t)\}_{t \in \mathbb{R}}$, respectively) and the sampled signal can be entirely recovered from the sample comb provided that the former is band limited, with bandwidth $2 B<v_{\mathrm{s}}=1 / T$. It suffices to filter the sample comb with a low-pass filter of cut-off frequency $B$.

Example 10. In Poisson sampling, the Bartlett spectrum of the sample comb is (in the density case)

$$
f_{Y}(v)=\lambda^{2} f_{X}(v)+\lambda \sigma_{X}^{2},
$$

where $\sigma_{X}^{2}=\operatorname{var}(X(t))$ is the power of the sampled signal. Therefore, whatever the sampling frequency $v_{\mathrm{s}}=\lambda$, there is no aliasing and the spectrum of the sampled signal can be recovered from that of the sample comb. However, if we apply the sample comb to a low-pass filter of cut-off frequency $v_{\mathrm{s}}=\lambda$, the output signal $\{Z(t)\}_{t \in \mathbb{R}}$ is a worse reconstruction of the sampled signal, which is assumed to be band limited with bandwidth $2 B$, in the sense that

$$
\mathrm{E}\left[|Z(t)-X(t)|^{2}\right]=\sigma_{X}^{2} .
$$

We will formulate random sampling in the general spatial case. Here, the sampled signal is a wide-sense stationary process $\{X(t)\}_{t \in \mathbb{R}^{m}}$ with mean $m_{X}$, autocovariance function $C_{X}(\tau)$, power spectral measure $\mu_{X}$, and Cramér-Khinchin decomposition $\left\{Z_{X}(A)\right\}_{A \subseteq B\left(\mathbb{R}^{m}\right)}$. Recall that the latter is a complex-valued stochastic process with centered, orthogonal increments, and that $\mathrm{E}\left[\left|Z_{X}(C)\right|^{2}\right]=\mu_{X}(C)$. Also, we have the Cramér-Khinchin decomposition formula

$$
X(t)=\int_{\mathbb{R}^{m}} \mathrm{e}^{2 \mathrm{i} \pi\langle v, t\rangle} Z_{X}(\mathrm{~d} \nu)+m_{X},
$$

in which the integral is of Wiener type. Note that, for all functions $g \in L_{\mathbb{C}}^{2}\left(\mu_{X}\right)$, the Wiener integral $\int_{\mathbb{R}^{m}} g(v) Z_{X}(\mathrm{~d} v)$ is well defined and is in $L_{\mathbb{C}}^{2}(P)$; moreover,

$$
\mathrm{E}\left[\left|\int_{\mathbb{R}^{m}} g(v) Z_{X}(\mathrm{~d} v)\right|^{2}\right]=\int_{\mathbb{R}^{m}}|g(v)|^{2} \mu_{X}(\mathrm{~d} v) .
$$


The sample 'brush'

$$
Y(t)=\sum_{s \in N} X(s) \delta(t-s)
$$

is identified with the measure

$$
\sum_{s \in N} X(s) \varepsilon_{S}(\cdot)
$$

We define the generalized Bochner spectrum of the sample brush to be a Radon measure $\mu_{Y}(\mathrm{~d} v)$ such that, for any $\varphi(t) \in B_{Y}$,

$$
\operatorname{var}\left(\int_{\mathbb{R}^{m}} \varphi(t) X(t) N(\mathrm{~d} t)\right)=\int_{\mathbb{R}^{m}}|\hat{\varphi}(\nu)|^{2} \mu_{Y}(\mathrm{~d} v),
$$

where $B_{Y}$ is a sufficiently large vector space of functions, here also called the 'test functions'. By 'sufficiently large', we mean that there cannot be two different Radon measures $\mu_{Y}$ satisfying (29) for all $\varphi \in B_{Y}$. Formally, since

$$
\begin{aligned}
\int_{\mathbb{R}^{m}} \varphi(t) Y(t) \mathrm{d} t & =\int_{\mathbb{R}^{m}} \varphi(t)\left(\sum_{s \in N} X(s) \delta(t-s)\right) \mathrm{d} t \\
& =\sum_{s \in N} \varphi(s) X(s) \\
& =\int_{\mathbb{R}^{m}} \varphi(t) X(t) N(\mathrm{~d} t),
\end{aligned}
$$

(29) becomes

$$
\operatorname{var}\left(\int_{\mathbb{R}^{m}} \varphi(t) Y(t) \mathrm{d} t\right)=\int_{\mathbb{R}^{m}}|\hat{\varphi}(v)|^{2} \mu_{Y}(\mathrm{~d} v) .
$$

Our next result concerns the case in which the sampler is independent of the signal. Let $N$ be a simple, wide-sense stationary point process on $\mathbb{R}^{m}$ with intensity $\lambda<\infty$, Bartlett spectrum $\mu_{N}$, and vector space of test functions $B_{N}$.

Theorem 5. Suppose that the sampled signal $\{X(t)\}$ and the point process $N$ are independent. Then the generalized process

$$
Y(t)=\sum_{s \in N} X(s) \delta(t-s)
$$

admits the extended Bochner power spectral measure

$$
\mu_{Y}=\mu_{N} * \mu_{X}+\lambda^{2} \mu_{X}+\left|m_{X}\right|^{2} \mu_{N} .
$$

If $B_{N}$ is stable with respect to multiplications by complex exponential functions, we can take $B_{Y}=B_{N}$.

Proof. By using (27), we have

$$
\begin{aligned}
\int_{\mathbb{R}^{m}} \varphi(t) X(t) N(\mathrm{~d} t) & =\int_{\mathbb{R}^{m}} \varphi(t)\left(\int_{\mathbb{R}^{m}} \mathrm{e}^{2 \mathrm{i} \pi\langle v, t\rangle} Z_{X}(\mathrm{~d} \nu)+m_{X}\right) N(\mathrm{~d} t) \\
& =\int_{\mathbb{R}^{m}}\left(\int_{\mathbb{R}^{m}} \varphi(t) \mathrm{e}^{2 \mathrm{i} \pi\langle v, t\rangle} N(\mathrm{~d} t)\right) Z_{X}(\mathrm{~d} \nu)+m_{X} \int_{\mathbb{R}^{m}} \varphi(t) N(\mathrm{~d} t),
\end{aligned}
$$


where we have formally exchanged the order of integration. Since the integrals with respect to $N(\mathrm{~d} t)$ and $Z_{X}(\mathrm{~d} \nu)$ are of different natures (one being an infinite sum, as usual, and the other a Wiener integral), this exchange must be formally justified, which we will do after completing the proof. By using the conditional variance formula, and defining $\mathcal{F}_{\infty}^{N}$ to be the sigma-field generated by $N$, we have

$$
\begin{aligned}
& \operatorname{var}\left(\int_{\mathbb{R}^{m}} \varphi(t) X(t) N(\mathrm{~d} t)\right) \\
& =\mathrm{E}\left[\operatorname{var}\left(\int_{\mathbb{R}^{m}}\left(\int_{\mathbb{R}^{m}} \varphi(t) \mathrm{e}^{2 \mathrm{i} \pi\langle v, t\rangle} N(\mathrm{~d} t)\right) Z_{X}(\mathrm{~d} \nu)+m_{X} \int_{\mathbb{R}^{m}} \varphi(t) N(\mathrm{~d} t) \mid \mathcal{F}_{\infty}^{N}\right)\right] \\
& \quad+\operatorname{var}\left(\mathrm{E}\left[\int_{\mathbb{R}^{m}}\left(\int_{\mathbb{R}^{m}} \varphi(t) \mathrm{e}^{2 \mathrm{i} \pi\langle v, t\rangle} N(\mathrm{~d} t)\right) Z_{X}(\mathrm{~d} v)+m_{X} \int_{\mathbb{R}^{m}} \varphi(t) N(\mathrm{~d} t) \mid \mathcal{F}_{\infty}^{N}\right]\right) \\
& =: \\
& \quad \alpha+\beta .
\end{aligned}
$$

Observe that, since $\varphi \in L^{2}\left(M_{2}\right)$,

$$
\left|\int_{\mathbb{R}^{m}} \varphi(t) \mathrm{e}^{2 \mathrm{i} \pi\langle v, t\rangle} N(\mathrm{~d} t)\right|^{2} \leq\left|\int_{\mathbb{R}^{m}}\right| \varphi(t)|N(\mathrm{~d} t)|^{2}<\infty \quad \text { almost surely. }
$$

Using the fact that when $N$ is fixed, $m_{X} \int_{\mathbb{R}^{m}} \varphi(t) N(\mathrm{~d} t)$ is deterministic, we obtain

$$
\begin{aligned}
\alpha & =\mathrm{E}\left[\operatorname{var}\left(\int_{\mathbb{R}^{m}}\left(\int_{\mathbb{R}^{m}} \varphi(t) \mathrm{e}^{2 \mathrm{i} \pi\langle v, t\rangle} N(\mathrm{~d} t)\right) Z_{X}(\mathrm{~d} v) \mid \mathcal{F}_{\infty}^{N}\right)\right] \\
& =\mathrm{E}\left[\int_{\mathbb{R}^{m}}\left|\int_{\mathbb{R}^{m}} \varphi(t) \mathrm{e}^{2 \mathrm{i} \pi\langle v, t\rangle} N(\mathrm{~d} t)\right|^{2} \mu_{X}(\mathrm{~d} v)\right] \quad(\text { by }(28) \text { and }(31)) \\
& =\int_{\mathbb{R}^{m}} \mathrm{E}\left[\left|\int_{\mathbb{R}^{m}} \varphi(t) \mathrm{e}^{2 \mathrm{i} \pi\langle v, t\rangle} N(\mathrm{~d} t)\right|^{2}\right] \mu_{X}(\mathrm{~d} v) \\
& =\int_{\mathbb{R}^{m}}\left(\operatorname{var}\left(\int_{\mathbb{R}^{m}} \varphi(t) \mathrm{e}^{2 \mathrm{i} \pi\langle v, t\rangle} N(\mathrm{~d} t)\right)+\left|\mathrm{E}\left[\int_{\mathbb{R}^{m}} \varphi(t) \mathrm{e}^{2 \mathrm{i} \pi\langle v, t\rangle} N(\mathrm{~d} t)\right]\right|^{2}\right) \mu_{X}(\mathrm{~d} v) \\
& =\int_{\mathbb{R}^{m}}\left(\int_{\mathbb{R}^{m}}|\hat{\varphi}(x-v)|^{2} \mu_{N}(\mathrm{~d} x)+\left|\int_{\mathbb{R}^{m}} \varphi(t) \mathrm{e}^{2 \mathrm{i} \pi\langle v, t\rangle} \lambda \mathrm{d} t\right|^{2}\right) \mu_{X}(\mathrm{~d} v) \\
& =\int_{\mathbb{R}^{m}}\left(\int_{\mathbb{R}^{m}}|\hat{\varphi}(x-v)|^{2} \mu_{N}(\mathrm{~d} x)\right) \mu_{X}(\mathrm{~d} v)+\lambda^{2} \int_{\mathbb{R}^{m}}|\hat{\varphi}(-v)|^{2} \mu_{X}(\mathrm{~d} v) \\
& =\int_{\mathbb{R}^{m}}\left(\int_{\mathbb{R}^{m}}|\hat{\varphi}(x+v)|^{2} \mu_{N}(\mathrm{~d} x)\right) \mu_{X}(\mathrm{~d} v)+\lambda^{2} \int_{\mathbb{R}^{m}}|\hat{\varphi}(v)|^{2} \mu_{X}(\mathrm{~d} v) \\
& =\int_{\mathbb{R}^{m}}|\hat{\varphi}(v)|^{2}\left(\mu_{N} * \mu_{X}\right)(\mathrm{d} v)+\lambda^{2} \int_{\mathbb{R}^{m}}|\hat{\varphi}(v)|^{2} \mu_{X}(\mathrm{~d} v)
\end{aligned}
$$

and, since

$$
\mathrm{E}\left[\int_{\mathbb{R}^{m}}\left(\int_{\mathbb{R}^{m}} \varphi(t) \mathrm{e}^{2 \mathrm{i} \pi\langle v, t\rangle} N(\mathrm{~d} t)\right) Z_{X}(\mathrm{~d} \nu) \mid \mathcal{F}_{\infty}^{N}\right]=0,
$$


we have

$$
\begin{aligned}
\beta & =\operatorname{var}\left(m_{X} \int_{\mathbb{R}^{m}} \varphi(t) N(\mathrm{~d} t)\right) \\
& =\left|m_{X}\right|^{2} \int_{\mathbb{R}^{m}}|\hat{\varphi}(v)|^{2} \mu_{N}(\mathrm{~d} v) \quad\left(\text { since } \varphi \in B_{N}\right) .
\end{aligned}
$$

Finally,

$$
\operatorname{var}\left(\int_{\mathbb{R}^{m}} \varphi(t) Y(t) \mathrm{d} t\right)=\int_{\mathbb{R}^{m}}|\hat{\varphi}(v)|^{2}\left(\mu_{N} * \mu_{X}+\lambda^{2} \mu_{X}+\left|m_{X}\right|^{2} \mu_{N}\right)(\mathrm{d} v),
$$

that is, $\{Y(t)\}_{t \in \mathbb{R}^{m}}$ admits the extended Bochner spectral measure given by (30).

It now remains to validate the exchange of integrals made at the start of the proof.

Lemma 3. Let $N$ be a simple, locally bounded stationary point process defined on $\mathbb{R}^{m}$ and admitting a Bartlett spectrum $\mu_{N}$. Let $M_{2}$ be its second moment measure and let $\{X(t)\}_{t \in \mathbb{R}^{m}}$ be a wide-sense stationary random field with Cramér-Khinchin decomposition $Z_{X}$ and power spectral measure $\mu_{X}$. Then, for all $\varphi \in L^{2}\left(M_{2}\right)$,

$$
\int_{\mathbb{R}^{m}} \varphi(t) X(t) N(\mathrm{~d} t)=\int_{\mathbb{R}^{m}}\left(\int_{\mathbb{R}^{m}} \varphi(t) \mathrm{e}^{2 \mathrm{i} \pi\langle v, t\rangle} N(\mathrm{~d} t)\right) Z_{X}(\mathrm{~d} v) .
$$

Proof. We provide the proof in the univariate case. The multivariate case follows along the same lines, with more notation. The left-hand side of (32) is

$$
A:=\sum_{n \in \mathbb{Z}} \varphi\left(T_{n}\right) X\left(T_{n}\right)=\lim _{c \uparrow \infty} \sum_{n \in \mathbb{Z}} \varphi\left(T_{n}\right) X\left(T_{n}\right) \mathbf{1}_{[-c, c]}\left(T_{n}\right)=\lim _{c \uparrow \infty} A(c),
$$

where the limit is in $L^{1}(P)$. Indeed

$$
\begin{aligned}
\mathrm{E}[|A-A(c)|] & \leq \mathrm{E}\left[\int_{\overline{[-c, c]}}|\varphi(t) X(t)| N(\mathrm{~d} t)\right] \\
& =\int_{\overline{[-c, c]}}|\varphi(t)| \mathrm{E}[|X(t)|] \lambda \mathrm{d} t \\
& \leq \lambda K \int_{\overline{[-c, c]}}|\varphi(t)| \mathrm{d} t,
\end{aligned}
$$

where

$$
K=\sup _{t} \mathrm{E}[|X(t)|]<\infty
$$

(by Schwarz's inequality, $\mathrm{E}[|X(t)|] \leq \mathrm{E}\left[|X(t)|^{2}\right]^{1 / 2}=\mathrm{E}\left[|X(0)|^{2}\right]^{1 / 2}$ ). Therefore, since $\varphi \in$ $L^{1}$, we have

$$
\lim _{c \uparrow \infty} \mathrm{E}[|A-A(c)|]=0 .
$$

The right-hand side of (32) is

$$
B:=\lim _{c \uparrow \infty} \int_{\mathbb{R}}\left(\int_{[-c, c]} \varphi(t) \mathrm{e}^{2 \mathrm{i} \pi\langle v, t\rangle} N(\mathrm{~d} t)\right) Z_{X}(\mathrm{~d} \nu)=\lim _{c \uparrow \infty} B(c),
$$


where the limit is in quadratic mean. Indeed,

$$
\begin{aligned}
\mathrm{E}\left[|B-B(c)|^{2}\right] & =\mathrm{E}\left[\left|\int_{\mathbb{R}}\left(\int_{\overline{[-c, c]}} \varphi(t) \mathrm{e}^{2 \mathrm{i} \pi v t} N(\mathrm{~d} t)\right) Z_{X}(\mathrm{~d} v)\right|^{2}\right] \\
& =\mathrm{E}\left[\mathrm{E}\left[\left|\int_{\mathbb{R}}\left(\int_{\overline{[-c, c]}} \varphi(t) \mathrm{e}^{2 \mathrm{i} \pi v t} N(\mathrm{~d} t)\right) Z_{X}(\mathrm{~d} v)\right|^{2} \mid \mathcal{F}_{\infty}^{N}\right]\right] \\
& =\mathrm{E}\left[\int_{\mathbb{R}}\left|\int_{\overline{[-c, c]}} \varphi(t) \mathrm{e}^{2 \mathrm{i} \pi v t} N(\mathrm{~d} t)\right|^{2} \mu_{X}(\mathrm{~d} v)\right] .
\end{aligned}
$$

Let $\varphi_{c}(t)=\varphi(t) \mathbf{1}_{\overline{[-c, c]}}(t)$. Then

$$
\mathrm{E}\left[\int_{\mathbb{R}}\left|\int_{\mathbb{R}} \varphi_{c}(t) \mathrm{e}^{2 \mathrm{i} \pi v t} N(\mathrm{~d} t)\right|^{2} \mu_{X}(\mathrm{~d} v)\right]=\int_{\mathbb{R}} \mathrm{E}\left[\left|\int_{\mathbb{R}} \varphi_{c}(t) \mathrm{e}^{2 \mathrm{i} \pi v t} N(\mathrm{~d} t)\right|^{2}\right] \mu_{X}(\mathrm{~d} v) .
$$

However,

$$
\begin{aligned}
\mathrm{E}\left[\left|\int_{\mathbb{R}} \varphi_{c}(t) \mathrm{e}^{2 \mathrm{i} \pi v t} N(\mathrm{~d} t)\right|^{2}\right] & \leq \mathrm{E}\left[\left(\int_{\mathbb{R}}\left|\varphi_{c}(t)\right| N(\mathrm{~d} t)\right)^{2}\right] \\
& =\int_{\mathbb{R} \times \mathbb{R}}\left|\varphi_{c}(t)\right|\left|\varphi_{c}(s)\right| M_{2}(\mathrm{~d} t \times \mathrm{d} s),
\end{aligned}
$$

a quantity that tends to 0 as $c \uparrow \infty$, by dominated convergence. Dominated convergence applied to the finite measure $\mu_{X}$ then yields the desired $L^{2}$-convergence. Furthermore,

$$
\begin{aligned}
A(c) & =\sum_{n \in \mathbb{Z}} \varphi\left(T_{n}\right) X\left(T_{n}\right) \mathbf{1}_{[-c, c]} \\
& =\sum_{n \in \mathbb{Z}} \varphi\left(T_{n}\right)\left(\int_{\mathbb{R}} \mathrm{e}^{2 \mathrm{i} \pi \nu T_{n}} Z_{X}(\mathrm{~d} \nu)\right) \mathbf{1}_{[-c, c]}\left(T_{n}\right) \\
& =\int_{\mathbb{R}}\left(\sum_{n \in \mathbb{Z}} \varphi\left(T_{n}\right) \mathrm{e}^{2 \mathrm{i} \pi \nu T_{n}} \mathbf{1}_{[-c, c]}\left(T_{n}\right)\right) Z_{X}(\mathrm{~d} \nu) \\
& =B(c),
\end{aligned}
$$

where we have used the fact that the sums involved are finite. Thus,

$$
\lim _{c \uparrow \infty} A(c)= \begin{cases}A & \text { in } L_{\mathbb{C}}^{1}(\mathrm{P}), \\ B & \text { in } L_{\mathbb{C}}^{2}(\mathrm{P}),\end{cases}
$$

from which it follows that $A=B$ almost surely. (To see this, we use the fact that if a sequence of random variables converges to some random variable in $L^{1}$ or $L^{2}$, then we can extract a subsequence that converges almost surely to the same random variable.)

Example 11. Let $N$ be a Cox process with a wide-sense stationary intensity $\{\lambda(t)\}_{t \in \mathbb{R}^{m}}$ with Bochner spectrum $\mu_{\lambda}$. Then

$$
\mu_{Y}=\mu_{\lambda} * \mu_{X}+\lambda^{2} \mu_{X}+\left|m_{X}\right|^{2} \mu_{\lambda}+\lambda \mathrm{E}\left[|X(0)|^{2}\right] \ell^{m},
$$

where, recall, $\ell^{m}$ is the Lebesgue measure. In particular, when the spike field is a homogeneous Poisson process,

$$
\mu_{Y}=\lambda^{2} \mu_{X}+\lambda \mathrm{E}\left[|X(0)|^{2}\right] \ell^{m} .
$$




\subsection{Reconstruction of the signal from its sampled version}

We now consider the problem of approximating $X(t)$ by a filtered version of $\{Y(t)\}$, namely

$$
\int_{\mathbb{R}^{m}} \varphi(t-s) Y(s) \mathrm{d} s,
$$

where $\varphi \in L^{1} \cap L^{2}$.

Moore and Thompson [22] gives motivation to this kind of problem in geophysics.

The difference between $X(t)$ and its approximation, that is, the reconstruction error, is measured by

$$
\epsilon=\mathrm{E}\left[\left|\int_{\mathbb{R}^{m}} \varphi(t-u) Y(u) \mathrm{d} u-X(t)\right|^{2}\right] .
$$

The following theorem gives another expression for the reconstruction error.

Theorem 6. Reconstruction of the signal $\{X(t)\}_{t \in \mathbb{R}}$ by filtering the sample comb $\{Y(t)\}_{t \in \mathbb{R}}$ with a filter $\varphi \in L^{1} \cap L^{2}$ gives the following error:

$\epsilon=\int_{\mathbb{R}^{m}}|\hat{\varphi}(v)|^{2} \mu_{Y}(\mathrm{~d} v)+\int_{\mathbb{R}^{m}} \mu_{X}(\mathrm{~d} v)-\lambda \int_{\mathbb{R}^{m}}\left(\hat{\varphi}(v)+\hat{\varphi}^{*}(v)\right) \mu_{X}(\mathrm{~d} v)+\left|m_{X}\right|^{2}|1-\lambda \hat{\varphi}(0)|^{2}$.

Proof. We have

$$
\begin{aligned}
\epsilon= & \mathrm{E}\left[\left|\int_{\mathbb{R}^{m}} \varphi(t-u) X(u) N(\mathrm{~d} u)-X(t)\right|^{2}\right] \\
= & \mathrm{E}\left[\left|\int_{\mathbb{R}^{m}} \varphi(t-u) X(u) N(\mathrm{~d} u)\right|^{2}\right]-2 \operatorname{Re}\left\{\mathrm{E}\left[\int_{\mathbb{R}} \varphi(t-u) X(t) X(u) N(\mathrm{~d} u)\right]\right\} \\
& +\mathrm{E}\left[|X(t)|^{2}\right] \\
= & : A-2 \operatorname{Re}\{B\}+C .
\end{aligned}
$$

In this expression,

$$
\begin{aligned}
A & =\int_{\mathbb{R}^{m}}|\hat{\varphi}(v)|^{2} \mu_{Y}(\mathrm{~d} v)+\lambda^{2}\left|m_{X}\right|^{2}\left|\int_{\mathbb{R}^{m}} \varphi(t) \mathrm{d} t\right|^{2} \\
& =\int_{\mathbb{R}^{m}}|\hat{\varphi}(v)|^{2} \mu_{Y}(\mathrm{~d} v)+\lambda^{2}\left|m_{X}\right|^{2}|\hat{\varphi}(0)|^{2} \\
B & =\mathrm{E}\left[\int_{\mathbb{R}^{m}} \varphi(t-u) X(t) X(u) N(\mathrm{~d} u)\right] \\
& =\lambda \int_{\mathbb{R}^{m}} \varphi(t-u) R_{X}(t-u) \mathrm{d} u \\
& =\lambda \int_{\mathbb{R}^{m}} \varphi(t) R_{X}(t) \mathrm{d} t \\
& =\lambda \int_{\mathbb{R}^{m}} \varphi(t) C_{X}(t) \mathrm{d} t+\lambda\left|m_{X}\right|^{2} \int_{\mathbb{R}^{m}} \varphi(t) \mathrm{d} t \\
& =\lambda \int_{\mathbb{R}^{m}} \hat{\varphi}(v) \mu_{X}(\mathrm{~d} v)+\lambda\left|m_{X}\right|^{2} \hat{\varphi}(0) \\
C & =\int_{\mathbb{R}^{m}} \mu_{X}(\mathrm{~d} v)+\left|m_{X}\right|^{2} .
\end{aligned}
$$


Therefore,

$$
\begin{aligned}
& \mathrm{E}\left[\left|\int_{\mathbb{R}^{m}} \varphi(t-u) X(u) N(\mathrm{~d} u)-X(t)\right|^{2}\right] \\
& \quad=\int_{\mathbb{R}^{m}}|\hat{\varphi}(v)|^{2} \mu_{Y}(\mathrm{~d} v)-\lambda \int_{\mathbb{R}^{m}}\left(\hat{\varphi}(v)+\hat{\varphi}(v)^{*}\right) \mu_{X}(\mathrm{~d} v) \\
& \quad+\left|m_{X}\right|^{2}\left(1-\lambda\left(\hat{\varphi}(0)+\hat{\varphi}(0)^{*}\right)+\lambda^{2}|\hat{\varphi}(0)|^{2}\right)+\int_{\mathbb{R}^{m}} \mu_{X}(\mathrm{~d} v) .
\end{aligned}
$$

In particular, in the case $m_{X}=0$ the error is

$$
\epsilon=\int_{\mathbb{R}^{m}}|\hat{\varphi}(v)|^{2} \mu_{Y}(\mathrm{~d} v)-2 \lambda \operatorname{Re}\left\{\int_{\mathbb{R}^{m}} \hat{\varphi}(v) \mu_{X}(\mathrm{~d} v)\right\}+\mu_{X}\left(\mathbb{R}^{m}\right) .
$$

We now give some examples of reconstruction error for different sampling schemes. For ease of notation, we assume that the signal is centered, that is, $m_{X}=0$. Moreover, some parts of the examples are developed in the univariate case. We perform the computations in the 'classical' situation of a band-limited signal $X(t)$ filtered with a band-limited (low-pass) filter $\varphi(v)$. More precisely, let $S$ be the support of $\mu_{X}$, with length $2 B=\ell(S)$. We then set

$$
\hat{\varphi}(v)= \begin{cases}1 / \lambda & \text { on } S \\ 0 & \text { otherwise }\end{cases}
$$

where $\lambda$ is the intensity of the spike comb.

Example 12. When $N$ is a homogeneous Poisson process with intensity $\lambda, \mu_{Y}$ is given by (33) and the error is

$$
\epsilon=\int_{\mathbb{R}^{m}}|\lambda \hat{\varphi}(v)-1|^{2} \mu_{X}(\mathrm{~d} v)+\lambda C_{X}(0) \int_{\mathbb{R}^{m}}|\hat{\varphi}(v)|^{2}(\mathrm{~d} v) .
$$

In the 'classical' band-limited case described above, we have

$$
\epsilon=\lambda C_{X}(0) \int_{\mathbb{R}}|\hat{\varphi}(v)|^{2}(\mathrm{~d} v)=\lambda C_{X}(0) \int_{\mathbb{R}} \frac{1}{\lambda^{2}} \mathbf{1}_{S}(v) \mathrm{d} v
$$

or, equivalently,

$$
\epsilon=C_{X}(0) \frac{2 B}{\lambda} .
$$

Therefore, sampling at the Nyquist rate $\lambda=2 B$ gives very poor performance, no better than the estimate based on no observations at all.

This does not mean, however, that below the rate $\lambda=2 B$ the samples contain no information (or, as the result suggests, 'negative information') about the process itself. A better choice of filter would give a linear estimate with error less than $\sigma^{2}=C_{X}(0)$. For instance, if we let $\hat{\varphi}$ be real, we find that

$$
\epsilon=\int_{\mathbb{R}}\left[(\lambda \hat{\varphi}(v)-1)^{2} f_{X}(v)+\lambda \sigma^{2} \hat{\varphi}(v)^{2}\right] \mathrm{d} \nu,
$$

where it is assumed that $\{X(t)\}_{t \in \mathbb{R}}$ has the power spectral density $f_{X}(v)$. The minimum error occurs for

$$
\hat{\varphi}(v)=\frac{\lambda f_{X}(v)}{\lambda^{2} f_{X}(v)+\lambda \sigma^{2}},
$$


and is

$$
\epsilon=\sigma^{2}\left(1-\int_{\mathbb{R}} \frac{\lambda \tilde{f}_{X}(v)}{1+\lambda \tilde{f}_{X}(v)} \tilde{f}_{X}(v) \mathrm{d} v\right),
$$

where $\tilde{f}_{X}(v)$ is the normalized power spectral density of $X(t)$ :

$$
\tilde{f}_{X}(v)=\frac{f_{X}(v)}{\int_{\mathbb{R}} f_{X}\left(v^{\prime}\right) \mathrm{d} v^{\prime}}=\frac{f_{X}(v)}{\sigma^{2}} .
$$

Therefore, $\epsilon=\sigma^{2}(1-\rho)$, where

$$
\rho=\int_{\mathbb{R}} \frac{\lambda \tilde{f}_{X}(\nu)}{1+\lambda \tilde{f}_{X}(v)} \tilde{f}_{X}(v) \mathrm{d} \nu,
$$

can be interpreted as the correlation coefficient between $X(t)$ and $N$, for fixed $t$.

Example 13. When the sampled comb is derived by $T$-uniform sampling, the reconstruction error (34) reads

$$
\begin{aligned}
\epsilon & =\frac{1}{T^{2}} \int_{\mathbb{R}}|\hat{\varphi}(v)|^{2} \mu_{X}(\mathrm{~d} v)-\frac{2}{T} \operatorname{Re}\left\{\int_{\mathbb{R}} \hat{\varphi}(v) \mu_{X}(\mathrm{~d} v)\right\}+\int_{\mathbb{R}} \mu_{X}(\mathrm{~d} v) \\
& =\int_{\mathbb{R}}\left|\frac{1}{T} \hat{\varphi}(v)-1\right|^{2} \mu_{X}(\mathrm{~d} v) .
\end{aligned}
$$

In the band-limited case, if we take $T=1 /(2 B)$, that is, $\lambda=2 B$, (35) gives an error equal to 0 . Therefore, the signal is perfectly reconstructed by

$$
X(t)=\int_{\mathbb{R}} \varphi(t-s) X(s) N(\mathrm{~d} s)=\sum_{n \in \mathbb{Z}} X\left(T_{n}\right) \operatorname{sinc}\left(t-T_{n}\right),
$$

where, here,

$$
\operatorname{sinc}(t)=\sin (2 \pi B t) /(2 \pi B t),
$$

which is the usual reconstruction formula (from the Shannon-Nyquist theorem).

Example 14. Given uniform samples in the presence of jitter, the reconstruction error is obtained by substituting the extended Bochner spectrum $\mu_{Y}$, with $\mu_{N}$ corresponding to a jittered uniform grid (see Example 1 and Corollary 2), into the error formula (34). The previous example showed that within the 'classical' sampling framework the signal may be perfectly reconstructed. In the presence of jitter, this is not possible and the reconstruction error is given by

$$
\epsilon=\frac{1}{2 B}\left(\int_{-B}^{B} \sigma^{2}\left(1-\left(\left|\psi_{Z}\right|^{2} * \tilde{f}_{X}\right)(v)\right) \mathrm{d} v\right) .
$$

\subsection{Signal-dependent rate of sampling}

We now consider the case in which the sampling rate depends on the process. The model for the sampler is now a Cox process [13] on $\mathbb{R}^{m}$ with an intensity of the form

$$
\lambda(t)=\lambda(t, X)
$$


that is conditional on $X$. For instance, in the univariate case,

$$
\lambda(t)=|X(t)|^{2} \quad \text { and } \quad \lambda(t)=|\dot{X}(t)|^{2},
$$

where $\dot{X}$ is the time derivative of $X(t)$, are possible intensities. More complicated functionals can be considered.

Theorem 7. Assume that

$$
\mathrm{E}\left[X(t)^{2} \lambda(t, X)^{2}\right]<\infty
$$

for all $t \in \mathbb{R}^{m}$, and that $\{\lambda(t)\}_{t \in \mathbb{R}^{m}}$ is a locally integrable process. Let $\mu_{Z}$ be the power spectrum of the stationary process

$$
Z(t)=X(t) \lambda(t)
$$

Then

$$
\mu_{Y}(\mathrm{~d} v)=\mu_{Z}(\mathrm{~d} v)+\overline{X^{2} \lambda} \mathrm{d} \nu
$$

where $\overline{X^{2} \lambda}=\mathrm{E}\left[X(t)^{2} \lambda(t)\right]$ (independent of $t$ ).

Proof. As in the independent case, in order to compute the Bartlett spectrum of $Y(t)$ we must evaluate the variance of

$$
\int_{\mathbb{R}^{m}} \varphi(t) Y(t) \mathrm{d} t=\int_{\mathbb{R}^{m}} \varphi(t) X(t) N(\mathrm{~d} t)
$$

for all $\varphi \in L^{1} \cap L^{2}$. We have

$$
\begin{aligned}
& \operatorname{var}\left(\int_{\mathbb{R}^{m}} \varphi(t) X(t) N(\mathrm{~d} t)\right) \\
& \quad=\mathrm{E}\left[\operatorname{var}\left(\int_{\mathbb{R}^{m}} \varphi(t) X(t) N(\mathrm{~d} t) \mid X\right)\right]+\operatorname{var}\left(\mathrm{E}\left[\int_{\mathbb{R}^{m}} \varphi(t) X(t) N(\mathrm{~d} t) \mid X\right]\right) \\
& \quad=\mathrm{E}\left[\int_{\mathbb{R}^{m}}|\varphi(t)|^{2}|X(t)|^{2} \lambda(t, X) \mathrm{d} t\right]+\operatorname{var}\left(\int_{\mathbb{R}^{m}} \varphi(t) X(t) \lambda(t, X) \mathrm{d} t\right)
\end{aligned}
$$

and, by definition of $\mu_{Z}$,

$$
\operatorname{var}\left(\int_{\mathbb{R}^{m}} \varphi(u) X(u) \lambda(u) \mathrm{d} u\right)=\int_{\mathbb{R}^{m}}|\hat{\varphi}(\nu)|^{2} \mu_{Z}(\mathrm{~d} \nu) .
$$

Therefore, recalling that $\mathrm{E}\left[X(t)^{2} \lambda(t)\right]=\overline{X^{2} \lambda}$ (independent of $t$ ), we have

$$
\begin{aligned}
\operatorname{var}\left(\int_{\mathbb{R}^{m}} \varphi(t) X(t) N(\mathrm{~d} t)\right) & =\operatorname{var}\left(\int_{\mathbb{R}^{m}} \varphi(u) X(u) \lambda(u) \mathrm{d} u\right)+\overline{X^{2} \lambda} \int_{\mathbb{R}^{m}}|\varphi(t)|^{2} \mathrm{~d} t \\
& =\int_{\mathbb{R}^{m}}|\hat{\varphi}(v)|^{2} \mu_{Z}(\mathrm{~d} v)+\overline{X^{2} \lambda} \int_{\mathbb{R}^{m}}|\hat{\varphi}(v)|^{2} \mathrm{~d} v \\
& =\int_{\mathbb{R}^{m}} \hat{\varphi}(v)\left(\mu_{Z}(\mathrm{~d} v)+\overline{X^{2} \lambda} \mathrm{d} v\right),
\end{aligned}
$$

and (36) follows. 
As particular cases of the above result, for $X(t) \equiv 1$ we recover the formula

$$
\mu_{N}(\mathrm{~d} v)=\mu_{\lambda}(\mathrm{d} v)+\lambda \mathrm{d} v
$$

for the Bartlett spectrum of the Cox process, and for $X(t)=\lambda(t)$ we have

$$
\mu_{Y}(\mathrm{~d} \nu)=\mu_{\lambda^{2}}(\mathrm{~d} \nu)+\mathrm{E}\left[\lambda^{3}(0)\right] \mathrm{d} \nu .
$$

\section{References}

[1] Abry, P. and Flandrin, P. (1996). Point processes, long-range dependence and wavelets. In Wavelets in Medicine and Biology, eds A. Aldroubi and M. Unser, CRC Press, Boca Raton, FL, pp. 413-437.

[2] Abry, P. And VeItch, D. (1998). Wavelet analysis of long-range-dependent traffic. IEEE Trans. Inf. Theory 44, $2-15$.

[3] Baccelli, F. ANd Blaszczyszyn, B. (2001). On a coverage process ranging from the Boolean model to the Poisson-Voronoi tessellation with applications to wireless communications. Adv. Appl. Prob. 33, 293-323.

[4] Beutler, F. J. and Leneman, O. A. Z. (1966). Random sampling of random processes. I. Stationary point processes. Inf. Control 9, 325-346.

[5] Beutler, F. J. and Leneman, O. A. Z. (1968). The spectral analysis of impulse processes. Inf. Control 12, 236-258.

[6] Bondesson, L. (1988). Shot-noise processes and shot-noise distributions. In Encyclopedia of Statistical Sciences, Vol. 8, John Wiley, New York, pp. 448-452.

[7] Brémaud, P. (2002). Mathematical Principles of Signal Processing. Fourier and Wavelet Analysis. Springer, New York.

[8] Brémaud, P. and Massoulié, L. (2001). Hawkes branching processes without ancestors. J. Appl. Prob. 38, 122-135.

[9] Brémaud, P. And Massoulié, L. (2002). Power spectra of general shot noises and Hawkes point processes with a random excitation. Adv. Appl. Prob. 34, 205-222.

[10] Brillinger, D. R. (1972). The spectral analysis of stationary interval functions. In Proc. Sixth Berkeley Symp. Math. Statist. Prob., Vol. 1, University of California Press, Berkeley, CA, pp. 483-513.

[11] Brillinger, D. R. (1981). Time Series. Data Analysis and Theory, 2nd edn. Holden-Day, Oakland, CA.

[12] Daley, D. J. (1970). Weakly stationary point processes and random measures. J. R. Statist. Soc. B 33, 406-428.

[13] Daley, D. J. And Vere-Jones, D. (1988). An Introduction to the Theory of Point Processes. Springer, New York.

[14] Daley, D. J. And Vere-Jones, D. (2002). An Introduction to the Theory of Point Processes, Vol. 1, Elementary Theory and Methods, 2nd edn. Springer, New York.

[15] Hawkes, A. G. (1971). Spectra of some self-exciting and mutually exciting point processes. Biometrika 58, 83-90.

[16] Hawkes, A. G. (1974). A cluster process representation of a self-exciting process. J. Appl. Prob. 11, $493-503$.

[17] Klüppelberg, C. And Mikosch, T. (1995). Explosive Poisson shot noise processes with applications to risk reserves. Bernoulli 1, 125-147.

[18] Leneman, O. A. Z. (1966). Random sampling of random processes. II. Impulse processes. Inf. Control 9, 347-363.

[19] Leneman, O. A. Z. ANd Lewis, J. B. (1966). Random sampling of random processes. Mean-square comparison of various interpolators. IEEE Trans. Automatic Control 11, 396-403.

[20] Masry, E. (1978). Alias-free sampling. An alternative conceptualization and its applications. IEEE Trans. Inf. Theory 24, 317-324.

[21] Masry, E. (1978). Poisson sampling and spectral estimation of continuous-time processes. IEEE Trans. Inf. Theory 24, 173-183.

[22] Moore, M. and Thompson, P. (1991). Impact of jittered sampling on conventional spectral estimates. $J$. Geophys. Res. 96, 18519-18526.

[23] Neveu, J. (1977). Processus ponctuels. In École d'Été de Probabilités de Saint-Flour VI (Lecture Notes Math. 598), Springer, Berlin, pp. 249-445.

[24] Ogata, Y. (1988). Statistical models for earthquake occurrence and residual analysis for point processes. $J$. Amer. Statist. Assoc. 83, 9-27.

[25] Ridolfi, A. (2004). Power spectra of random spikes and related complex signals, with application to communications. Doctoral Thesis. École Polytechnique Fédérale de Lausanne. Available at http://infoscience. epfl.ch/search.py?recid=33626\&ln=en.

[26] Ridolfi, A. ANd Win, M. Z. (2005). Power spectra of multipath faded pulse trains. In Proc. IEEE Internat. Symp. Inf. Theory, IEEE, pp. 102-106. 
[27] Ridolfi, A. AND Win, M. Z. (2005). Ultrawide bandwidth signals as shot-noise: a unifying approach. To appear in IEEE J. Select. Areas Commun. (special issue on Ultra-Wideband Wireless Communications: Theory and Applications).

[28] Samorodnitsky, G. (1995). A class of shot noise models for financial applications. In Athens Conf. Appl. Prob. Time Series Anal. (Lecture Notes Statist. 114), Vol. 1, eds C. C. Heyde et al., Springer, New York, pp. 332-353.

[29] Ссноттку, W. (1918). Über spontane Stromschwankungen in verschiedenen Elektrizitätsleitern. Ann. Physik 57, 541-567.

[30] Shapiro, H. S. and Silverman, R. A. (1960). Alias-free sampling of random noise. J. Soc. Indust. Appl. Math. 8, 225-248.

[31] Vere-Jones, D. And Davies, R. B. (1966). A statistical survey of earthquakes in the main seismic area of New Zealand. Part II: Time series analysis. N. Z. J. Geol. Geophys. 9, 251-284. 